\title{
Extreme mass ratio inspirals on the equatorial plane in the adiabatic order
}

\author{
Ryuichi Fujita $^{1,2}$ and Masaru Shibata ${ }^{3,1}$ \\ ${ }^{1}$ Center for Gravitational Physics, Yukawa Institute for Theoretical Physics, Kyoto University, Kyoto 606-8502, Japan \\ ${ }^{2}$ Institute of Liberal Arts, Otemon Gakuin University, Osaka 567-8502, Japan \\ ${ }^{3}$ Max Planck Institute for Gravitational Physics (Albert Einstein Institute), \\ Am Mhlenberg 1, Potsdam-Golm 14476, Germany
}

(Dated: September 10, 2020)

\begin{abstract}
We compute gravitational waves from inspiraling stellar-mass compact objects on the equatorial plane of a massive spinning black hole $(\mathrm{BH})$. Our inspiral orbits are computed by taking into account the adiabatic change of orbital parameters due to gravitational radiation in the lowest order in mass ratio. We employ an interpolation method to compute the adiabatic change at arbitrary points inside the region of orbital parameter space computed in advance. Using the obtained inspiral orbits and associated gravitational waves, we compute power spectra of gravitational waves and the signal-to-noise ratio (SNR) for several values of the BH spin, the masses of the binary, and the initial orbital eccentricity during a hypothetical three-year Laser Interferometer Space Antenna observation before final plunge. We find that (i) the SNR increases as the BH spin and the mass of the compact object increase for the $\mathrm{BH}$ mass $M \gtrsim 10^{6} M_{\odot}$, (ii) the $\mathrm{SNR}$ has a maximum for $M \approx 10^{6} M_{\odot}$, and (iii) the SNR increases as the initial eccentricity increases for $M=10^{6} M_{\odot}$. We also show that incorporating the contribution from the higher multipole modes of gravitational waves is crucial for enhancing the detection rate.
\end{abstract}

\section{INTRODUCTION}

The inspirals of stellar-mass compact objects of mass $\mu \sim 1-100 M_{\odot}$ into supermassive black holes (SMBHs) of mass $M \sim 10^{5}-10^{7} M_{\odot}$ are among the key sources for the future space-based gravitational-wave detector Laser Interferometer Space Antenna (LISA) [1] (see, e.g., Refs. [2, 3] for other future space-based detectors in the LISA band). Such extreme-mass-ratio inspirals (EMRIs) are expected to have typically $\sim 10^{5}$ orbital cycles $\left(\sim 10^{6} \mathrm{rad}\right.$ in gravitational-wave phase) during a few years of observation by LISA. The observation of gravitational waves from EMRIs will provide an opportunity of precision probes of general relativity and information in the vicinity of SMBHs (see, e.g., Refs. [4, 5]). However, for these research purposes, one has to prepare accurate models of gravitational waveforms suitable for the data analysis of gravitational waves from EMRIs.

Since the mass ratio $\eta \equiv \mu / M$ is $\lesssim 10^{-3}$, EMRIs can be modeled by using black hole $(\mathrm{BH})$ perturbation theory (see, e.g., Refs. $[6,7]$ ). In the limit of the test mass, $\eta \rightarrow 0$, the compact object follows timelike geodesic orbits in background Kerr spacetime. At higher order in the mass ratio, however, the orbit deviates slightly from geodesic orbits due to the interaction with its own gravitational field, gravitational self-force (GSF) (see, e.g., Refs. [8-12] and references therein). Using the two-timescale expansion method in Ref. [13], the orbital phase can be expanded with respect to $\eta$ as

$$
\Phi=\frac{1}{\eta}\left(\Phi^{(0)}+\eta \Phi^{(1)}+\mathcal{O}\left(\eta^{2}\right)\right),
$$

where $\Phi^{(0)}$ and $\Phi^{(1)}$ are quantities of order unity, and resonances [14] of $\mathcal{O}\left(\eta^{1 / 2}\right)$ are neglected. $\Phi^{(0)} / \eta$ denotes the orbital phase determined by the time-averaged dissipative part of the first-order GSF, that corresponds to the adiabatic change of the constants of motion of the geodesics. $\Phi^{(1)}$ denotes the remaining parts of the selfforce. $\Phi^{(0)}$ must be computed much more precisely than any others because $\Phi^{(0)} / \eta$ is the dominant part of the orbital phase.

In order to determine $\Phi^{(0)}$, one has to compute orbital inspirals by incorporating the adiabatic change of the constants of motion, $d I^{i} / d t$, due to the gravitationalwave emission, where $I^{i}$ denotes three constants of motion (see Sec. II for details). Many numerical results of $d I^{i} / d t$ have been derived for spherical orbits [15-17], eccentric-equatorial orbits [18-20], and eccentric-inclined orbits [21-23]. The orbital phase $\Phi^{(0)} / \eta$ is also computed for spherical orbits in Ref. [17], but the accuracy in $\Phi^{(0)} / \eta$ is worse than $1 \mathrm{rad}$, which is the minimum accuracy required for the gravitational-wave modeling suitable for the data analysis of EMRIs.

In this paper, we derive adiabatic orbital inspirals on the equatorial plane of the Kerr BH focusing on the timeaveraged dissipative part of the first-order GSF (i.e., the lowest-order part in $\eta$ ). We compute the adiabatic evolution of the inspiral orbits using the osculating geodesics method [24, 25], in which a sequence of geodesic orbits is assumed to be tangent to the true inspiral orbit at each moment (see Refs. [26, 27] for inspiral orbits including the conservative part of the first-order GSF in Schwarzschild spacetime). Our adiabatic inspiral orbits are computed by taking into account the adiabatic change of the constants of motion due to the emission of gravitational waves at each geodesic orbit.

The issue in this line of the study is that numerical computation for the adiabatic change of $I^{i}$ for each of $\sim$ $10^{5}$ inspiral orbits is extremely costly, even if we restrict our attention to equatorial inspirals. Thus, we employ the following alternative strategy. First, we compute the adiabatic change of $I^{i}$ for a number of data points in 
the semilatus rectum, $p$, and the orbital eccentricity, $e$. Then, we use an interpolation method to obtain $d I^{i} / d t$ at arbitrary points in the phase space of $(p, e)$, for which $d I^{i} / d t$ are computed in advance. Using this strategy, we obtain inspiral orbits and associated gravitational waves with an inexpensive computational cost.

This paper is organized as follows. In Sec. II, we review formulations necessary to compute the geodesic motion in Kerr spacetime and the adiabatic change of the constants of motion due to the emission of gravitational waves. In Sec. III, we first summarize our approach to obtain the adiabatic inspiral orbits using the osculating geodesic method with interpolated fluxes. Then, we describe the accuracy for the adiabatic change of constants of motion numerically derived. The issues to improve the accuracy are also discussed. Several representative inspiral orbits and associated gravitational waves are presented in Sec. IV, paying particular attention to gravitational-wave spectra. We show the dependence of the gravitational-wave spectra on the mass and spin of SMBHs and orbital eccentricity of compact objects. We summarize this paper in Sec. V. Throughout this paper we use the geometrical units with $c=G=1$ where $c$ and $G$ are the speed of light and gravitational constant, respectively.

\section{FORMULATION}

The purpose of this paper is to explore inspiral orbits of a stellar-mass compact object of mass $\mu$ around a Kerr $\mathrm{BH}$ of mass $M \gg \mu$. Specifically, we derive gravitational waves emitted by the orbiting object using the $\mathrm{BH}$ perturbation theory and consider the adiabatic evolution of the orbit due to the gravitational-wave emission. We use the methods presented in Refs. [23, 28, 29], based on the formalism developed in Refs. [7, 30-32], to numerically compute gravitational-wave fluxes by a stellar-mass object with bound orbits around a Kerr BH of spin $a$ for large sets of orbital parameters. Then, one can obtain inspiral orbits by incorporating the adiabatic change of orbital parameters due to the gravitational-wave emission. In this paper, we focus only on the inspirals on the equatorial plane of the $\mathrm{BH}$ as a first step.

\section{A. Bound geodesics}

First, we summarize the method to determine the generic geodesic orbit in Kerr spacetime. Using BoyerLindquist coordinates for the Kerr solution, $(t, r, \theta, \phi)$, and Mino time [33] $\lambda=\int d \tau /\left(r^{2}+a^{2} \cos ^{2} \theta\right)$, the geodesic equations are written as

$$
\begin{gathered}
\left(\frac{d r}{d \lambda}\right)^{2}=R(r), \\
\left(\frac{d \cos \theta}{d \lambda}\right)^{2}=\Theta(\cos \theta), \\
\frac{d \phi}{d \lambda}=\Phi_{r}(r)+\Phi_{\theta}(\theta), \\
\frac{d t}{d \lambda}=T_{r}(r)+T_{\theta}(\theta),
\end{gathered}
$$

where

$$
\begin{aligned}
R(r)= & {[P(r)]^{2}-\Delta\left[r^{2}+\left(\mathcal{L}_{z}-a \mathcal{E}\right)^{2}+\mathcal{C}\right], } \\
P(r)= & \left(r^{2}+a^{2}\right) \mathcal{E}-a \mathcal{L}_{z} \\
\Theta(\cos \theta)= & \mathcal{C}-\left(\mathcal{C}+a^{2}\left(1-\mathcal{E}^{2}\right)+\mathcal{L}_{z}^{2}\right) \cos ^{2} \theta \\
& +a^{2}\left(1-\mathcal{E}^{2}\right) \cos ^{4} \theta \\
\Phi_{r}(r)= & \frac{a}{\Delta} P(r), \\
\Phi_{\theta}(\theta)= & \frac{\mathcal{L}_{z}}{1-\cos ^{2} \theta}-a \mathcal{E} \\
T_{r}(r)= & \frac{r^{2}+a^{2}}{\Delta} P(r) \\
T_{\theta}(\theta)= & -a^{2} \mathcal{E}\left(1-\cos ^{2} \theta\right)+a \mathcal{L}_{z},
\end{aligned}
$$

and $\Delta=r^{2}-2 M r+a^{2} . \mathcal{E}, \mathcal{L}_{z}$, and $\mathcal{C}$ are constants that denote the specific energy, the $z$ component of the specific angular momentum, and the Carter constant of a stellar-mass compact object, respectively. The geodesic orbits in Kerr spacetime can be characterized by these three constants of motion $\left(\mathcal{E}, \mathcal{L}_{z}, \mathcal{C}\right)$. In the following, we often refer to these three constants in a vector form as $I^{i}$.

One can also use another set of three parameters, the semilatus rectum $p$, the orbital eccentricity $e$, and the inclination angle $\theta_{\text {inc }}$, to characterize the geodesics for bound orbits. These parameters are related to turning points of the radial motion, $r_{\max }$ and $r_{\min }$, and the polar motion, $\theta_{\min }$, via

$$
p=\frac{2 r_{\max } r_{\min }}{r_{\max }+r_{\min }}, \quad e=\frac{r_{\max }-r_{\min }}{r_{\max }+r_{\min }},
$$

and $\theta_{\text {inc }}=\pi / 2-\left(\operatorname{sgn} \mathcal{L}_{z}\right) \theta_{\min }$. Note that $r_{\min }$ is written as $p /(1+e)$ and the minimum value of $r_{\min }$ is written as $2 M-a+2 M^{1 / 2} \sqrt{M-a}$, which is realized for the marginally bound orbit [34]. $\left(\mathcal{E}, \mathcal{L}_{z}, \mathcal{C}\right)$ are written as functions of $\left(p, e, \theta_{\text {inc }}\right)$ using the method in Ref. [35]. In the following, we refer to $\left(p, e, \theta_{\text {inc }}\right)$ as $J^{i}$.

Using $J^{i}$, the radial and polar motions can be parametrized by

$$
\begin{aligned}
r(\lambda) & =\frac{p}{1+e \cos \left[\chi(\lambda)-\chi_{0}\right]}, \\
\cos \theta(\lambda) & =\cos \theta_{\text {inc }} \cos \left[\psi(\lambda)-\psi_{0}\right],
\end{aligned}
$$


where $\chi(\lambda)$ and $\psi(\lambda)$ are monotonic parameters that run from 0 to $2 \pi$ over one radial and polar cycle, respectively. $\chi_{0}$ and $\psi_{0}$ take the values of $\chi$ and $\psi$ at $r=r_{\min }$ and $\theta=$ $\theta_{\min }$, respectively. In the osculating geodesic method, the inspiral orbit under the GSF is described by the evolution of $p, e, \theta_{\text {inc }}, \chi_{0}$, and $\psi_{0}$. The principal orbital elements $p$, $e$, and $\theta_{\text {inc }}$ evolve due to the dissipative part of the GSF, while the positional orbital elements $\chi_{0}$ and $\psi_{0}$ evolve due to the conservative part of the GSF.

Since the equations of radial and polar motion are decoupled in Eqs. (2) and (3), for the bound orbits, $r(\lambda)$ and $\theta(\lambda)$ become periodic functions that are independent of each other. The fundamental periods for the radial and polar motion, $\Lambda_{r}$ and $\Lambda_{\theta}$, are calculated by

$$
\Lambda_{r}=2 \int_{\mathrm{r}_{\min }}^{\mathrm{r}_{\max }} \frac{d r}{\sqrt{R(r)}}, \quad \Lambda_{\theta}=4 \int_{0}^{\cos \theta_{\min }} \frac{d \cos \theta}{\sqrt{\Theta(\cos \theta)}},
$$

and thus, the angular frequencies of the radial and polar motion become

$$
\Upsilon_{r}=\frac{2 \pi}{\Lambda_{r}}, \quad \Upsilon_{\theta}=\frac{2 \pi}{\Lambda_{\theta}}
$$

$\Upsilon_{r}$ and $\Upsilon_{\theta}$ can be expressed in complete elliptic integrals of the first kind; see, e.g., Refs. [23, 36].

We define the angle variables as $w_{r}=\Upsilon_{r} \lambda$ and $w_{\theta}=$ $\Upsilon_{\theta} \lambda$. Then, any functions that depend only on $r$ or $\theta$ become periodic with respect to $w_{r}$ or $w_{\theta}$, respectively, with the period of $2 \pi$.

To solve Eqs. (4) and (5), we expand their right-hand sides into Fourier series as [37]

$$
\begin{aligned}
& \frac{d t}{d \lambda}=\sum_{k, n} T_{k, n} e^{-i k \Upsilon_{r} \lambda} e^{-i n \Upsilon_{\theta} \lambda}, \\
& \frac{d \phi}{d \lambda}=\sum_{k, n} \Phi_{k, n} e^{-i k \Upsilon_{r} \lambda} e^{-i n \Upsilon_{\theta} \lambda}
\end{aligned}
$$

where

$$
\begin{aligned}
T_{k, n} & =\int_{0}^{2 \pi} \frac{d w_{r}}{2 \pi} \int_{0}^{2 \pi} \frac{d w_{\theta}}{2 \pi}\left(T_{r}(r)+T_{\theta}(\theta)\right) e^{i k w_{r}} e^{i n w_{\theta}} \\
\Phi_{k, n} & =\int_{0}^{2 \pi} \frac{d w_{r}}{2 \pi} \int_{0}^{2 \pi} \frac{d w_{\theta}}{2 \pi}\left(\Phi_{r}(r)+\Phi_{\theta}(\theta)\right) e^{i k w_{r}} e^{i n w_{\theta}}
\end{aligned}
$$

Since $T_{k, n}=0$ and $\Phi_{k, n}=0$ for $k \neq 0$ and $n \neq 0$, we have

$$
\begin{aligned}
& \frac{d t}{d \lambda}=\Gamma+\sum_{k \neq 0} T_{k, 0} e^{-i k w_{r}}+\sum_{n \neq 0} T_{0, n} e^{-i n w_{\theta}} \\
& \frac{d \phi}{d \lambda}=\Upsilon_{\phi}+\sum_{k \neq 0} \Phi_{k, 0} e^{-i k w_{r}}+\sum_{n \neq 0} \Phi_{0, n} e^{-i n w_{\theta}}
\end{aligned}
$$

where

$$
\begin{aligned}
\Gamma & \equiv T_{00}=\Upsilon_{t^{(r)}}+\Upsilon_{t^{(\theta)}}, \\
\Upsilon_{\phi} & \equiv \Phi_{00}=\Upsilon_{\phi^{(r)}}+\Upsilon_{\phi^{(\theta)}}, \\
\Upsilon_{t^{(r)}} & =\frac{1}{2 \pi} \int_{0}^{2 \pi} d w_{r} T_{r}, \Upsilon_{t^{(\theta)}}=\frac{1}{2 \pi} \int_{0}^{2 \pi} d w_{\theta} T_{\theta}, \\
\Upsilon_{\phi^{(r)}} & =\frac{1}{2 \pi} \int_{0}^{2 \pi} d w_{r} \Phi_{r}, \Upsilon_{\phi^{(\theta)}}=\frac{1}{2 \pi} \int_{0}^{2 \pi} d w_{\theta} \Phi_{\theta}
\end{aligned}
$$

Then, we obtain the functions $t(\lambda)$ and $\phi(\lambda)$ by integrating Eqs. (21) and (22) in the following forms:

$$
\begin{aligned}
t(\lambda) & =\Gamma \lambda+\sum_{k \neq 0} \frac{i T_{k, 0}}{k \Upsilon_{r}} e^{-i k w_{r}}+\sum_{n \neq 0} \frac{i T_{0, n}}{n \Upsilon_{\theta}} e^{-i n w_{\theta}} \\
\phi(\lambda) & =\Upsilon_{\phi} \lambda+\sum_{k \neq 0} \frac{i \Phi_{k, 0}}{k \Upsilon_{r}} e^{-i k w_{r}}+\sum_{n \neq 0} \frac{i \Phi_{0, n}}{n \Upsilon_{\theta}} e^{-i n w_{\theta}}
\end{aligned}
$$

Here, the two variables, $\Gamma$ and $\Upsilon_{\phi}$, denote the average rates of change of $t$ and $\phi$ as functions of $\lambda$, respectively.

\section{B. Secular evolution of orbital parameters}

In the Teukolsky formalism [38], the gravitational perturbation on Kerr spacetime is described in terms of the Newman-Penrose variables, $\Psi_{0}$ and $\Psi_{4}$, which satisfy a master equation. The Weyl scalar $\Psi_{4}$ is related to gravitational waves at infinity as

$$
\Psi_{4} \rightarrow \frac{1}{2}\left(\ddot{h}_{+}-i \ddot{h}_{\times}\right)
$$

The master equation for $\Psi_{4}$ can be separated into radial and angular parts if we expand $\Psi_{4}$ in harmonic modes as

$$
\rho^{-4} \Psi_{4}=\sum_{\ell m} \int_{-\infty}^{\infty} d \omega e^{-i \omega t+i m \varphi}{ }_{-2} S_{\ell m}^{a \omega}(\theta) R_{\ell m \omega}(r),
$$

where $\rho=(r-i a \cos \theta)^{-1}$, and ${ }_{-2} S_{\ell m}^{a \omega}(\theta)$ is the spinweighted spheroidal harmonics with spin $s=-2$. The radial function $R_{\ell m \omega}(r)$ satisfies the so-called Teukolsky equation,

$$
\Delta^{2} \frac{d}{d r}\left(\frac{1}{\Delta} \frac{d R_{\ell m \omega}}{d r}\right)-V(r) R_{\ell m \omega}=T_{\ell m \omega},
$$

where the potential term $V(r)$ is

$$
V(r)=-\frac{K^{2}+4 i(r-M) K}{\Delta}+8 i \omega r+\lambda_{\ell m \omega},
$$

with $K=\left(r^{2}+a^{2}\right) \omega-m a$ and $\lambda_{\ell m \omega}$ the eigenvalue of ${ }_{-2} S_{\ell m}^{a \omega}(\theta)$.

The asymptotic behavior of the solution at the horizon and infinity is written, respectively, as

$$
R_{\ell m \omega}\left(r \rightarrow r_{+}\right) \equiv Z_{\ell m \omega}^{\mathrm{H}} \Delta^{2} e^{-i P r^{*}},
$$


and

$$
R_{\ell m \omega}(r \rightarrow \infty) \equiv Z_{\ell m \omega}^{\infty} r^{3} e^{i \omega r^{*}}
$$

where $r_{+}=M+\sqrt{M^{2}-a^{2}}, P=\omega-m a / 2 M r_{+}$, and $r^{*}$ is the tortoise coordinate.

For the bound orbits of a stellar-mass object, the amplitude of the partial wave $Z_{\ell m \omega}^{\infty / \mathrm{H}}$, defined in Eqs. (30) and (31), can be expanded as

$$
Z_{\ell m \omega}^{\infty, \mathrm{H}} \equiv \sum_{k n} \tilde{Z}_{\ell m k n}^{\infty, \mathrm{H}} \delta\left(\omega-\omega_{m k n}\right)
$$

where

$$
\omega_{m k n} \equiv\left(m \Upsilon_{\phi}+k \Upsilon_{\theta}+n \Upsilon_{r}\right) / \Gamma
$$

Using these functions, gravitational waves at infinity are expressed as

$h_{+}-i h_{\times}=-\frac{2}{r} \sum_{\ell m k n} \frac{\tilde{Z}_{\ell m k n}^{\infty}}{\omega_{m k n}^{2}} \frac{-2 S_{\ell m}^{a \omega_{m k n}}(\theta)}{\sqrt{2 \pi}} e^{i \omega_{m k n}\left(r^{*}-t\right)+i m \phi}$.

In addition, the adiabatic change for $\left(\mathcal{E}, \mathcal{L}_{z}, \mathcal{C}\right)$ due to the emission of gravitational waves are expressed as [33, 39, 40]

$$
\begin{aligned}
\left\langle\frac{d \mathcal{E}}{d t}\right\rangle & =-\mu^{2} \sum_{\ell m k n} \frac{1}{4 \pi \omega_{m k n}^{2}}\left(\left|\tilde{Z}_{\ell m k n}^{\infty}\right|^{2}+\alpha_{\ell m k n}\left|\tilde{Z}_{\ell m k n}^{\mathrm{H}}\right|^{2}\right), \\
\left\langle\frac{d \mathcal{L}_{z}}{d t}\right\rangle & =-\mu^{2} \sum_{\ell m k n} \frac{m}{4 \pi \omega_{m k n}^{3}}\left(\left|\tilde{Z}_{\ell m k n}^{\infty}\right|^{2}+\alpha_{\ell m k n}\left|\tilde{Z}_{\ell m k n}^{\mathrm{H}}\right|^{2}\right) \\
\left\langle\frac{d \mathcal{C}}{d t}\right\rangle & =\left\langle\frac{d \mathcal{Q}}{d t}\right\rangle-2\left(a \mathcal{E}-\mathcal{L}_{z}\right)\left(a\left\langle\frac{d \mathcal{E}}{d t}\right\rangle-\left\langle\frac{d \mathcal{L}_{z}}{d t}\right\rangle\right), \\
\left\langle\frac{d \mathcal{Q}}{d t}\right\rangle & =2 \Upsilon_{t^{(r)}}\left\langle\frac{d \mathcal{E}}{d t}\right\rangle-2 \Upsilon_{\phi^{(r)}}\left\langle\frac{d \mathcal{L}_{z}}{d t}\right\rangle \\
& +\mu^{3} \sum_{\ell m k n} \frac{n \Upsilon_{r}}{2 \pi \omega_{m k n}^{3}}\left(\left|\tilde{Z}_{\ell m k n}^{\infty}\right|^{2}+\alpha_{\ell m k n}\left|\tilde{Z}_{\ell m k n}^{\mathrm{H}}\right|^{2}\right),
\end{aligned}
$$

where

$$
\alpha_{\ell m k n}=\frac{256\left(2 M r_{+}\right)^{5} P\left(P^{2}+4 \epsilon^{2}\right)\left(P^{2}+16 \epsilon^{2}\right) \omega_{m k n}^{3}}{C_{\ell m k n}^{\mathrm{TS}}},
$$

$\epsilon=\sqrt{M^{2}-a^{2}} / 4 M r_{+}$, and $C_{\ell m k n}^{\mathrm{TS}}$ is the TeukolskyStarobinsky constant [41] (see Ref. [42] for the scalar case). Here $\langle\cdots\rangle$ denotes the time average. We note that $\tilde{Z}_{\ell m k n}^{\infty}$ and $\tilde{Z}_{\ell m k n}^{\mathrm{H}}$ in Eqs. (35)-(38) denote fluxes at infinity and the horizon, respectively.
Once we obtain the adiabatic change of $I^{i}$, we can derive the adiabatic change of $J^{i}$ using

$$
\left\langle\frac{d J^{i}}{d t}\right\rangle=\left(G^{-1}\right)_{j}^{i}\left\langle\frac{d I^{j}}{d t}\right\rangle
$$

where $G_{j}^{i}=\partial I^{i} / \partial J^{j}$. In this paper, we consider the case of $\mathcal{C}=0$, and thus, $d \mathcal{C} / d t=0\left(d \theta_{\text {inc }} / d t=0\right)$.

\section{OUR METHOD TO OBTAIN INSPIRAL ORBITS}

The purpose of this paper is to derive adiabatic inspiral orbits and associated gravitational waves. We ignore the change in the mass and spin of the $\mathrm{BH}$ due to the absorption of gravitational waves because they are small effects. In order to obtain the adiabatic inspiral orbits, we construct a sequence of the osculating orbits [24, 25], which are assumed to be tangent to the true inspiral orbit at each instance. We ignore the evolution of the positional orbital elements, which is one of the higher-order effects in the mass ratio (see Refs. [24, 25] for a method to include the evolution of the positional orbital elements). We incorporate the adiabatic change of the constants of motion due to the emission of gravitational waves for each geodesic orbit. Then, the error in our inspiral orbit from the true inspiral orbit is of $\mathcal{O}(\eta)$, which is caused by higher-order effects ignored in this paper. The evolution of $(p, e)$ is calculated by determining $(\langle d p / d t\rangle,\langle d e / d t\rangle)$ ,from Eq. (40) for fixed values of $M$ and $q(=a / M)$ : dimensionless spin parameter. In the following we refer to $q$ simply as the $\mathrm{BH}$ spin.

\section{A. Procedure for determining inspiral orbits}

For the numerical evolution of $p$, i.e., $p(t)$, using interpolated gravitational-wave fluxes, we take a Euler step as $p(t+\Delta t)=p(t)+\langle d p / d t\rangle \Delta t$, where $\Delta t$ is a time step and $\langle d p / d t\rangle$ is computed from Eq. (40). In this paper, we choose $\Delta t=p /\langle d p / d t\rangle \epsilon_{t}$, where $\epsilon_{t} \approx 10^{-4}$. In order to estimate the relative error of $p(t)$, we compare $p(t)$ by setting $\epsilon_{t}=10^{-4}$ with a reference solution for $p(t)$ obtained by setting $\epsilon_{t}=10^{-6}$. We find that the relative error in $p(t)$ by setting $\epsilon_{t}=10^{-4}$ is about $\mathcal{O}\left(\epsilon_{t}\right)$, i.e., $10^{-4}$. In Sec. IV, we find that the power spectra and the signalto-noise ratio (SNR) for typical EMRIs span about a few orders of magnitude. The error of $10^{-4}$ in the inspiral orbits is acceptable for computing power spectra of gravitational waves and the SNR within the error of $10^{-3}$, although the error size in the adiabatic change needs to be better than $10^{-6}$ to suppress the error in the total orbital phase less than $1 \mathrm{rad}$ (see discussion below). We note that the above procedure can be straightforwardly extended to the higher-order $\mathrm{BH}$ perturbation theory in $\eta$.

It is feasible to numerically calculate the adiabatic change of the constants of motion only for a restricted 

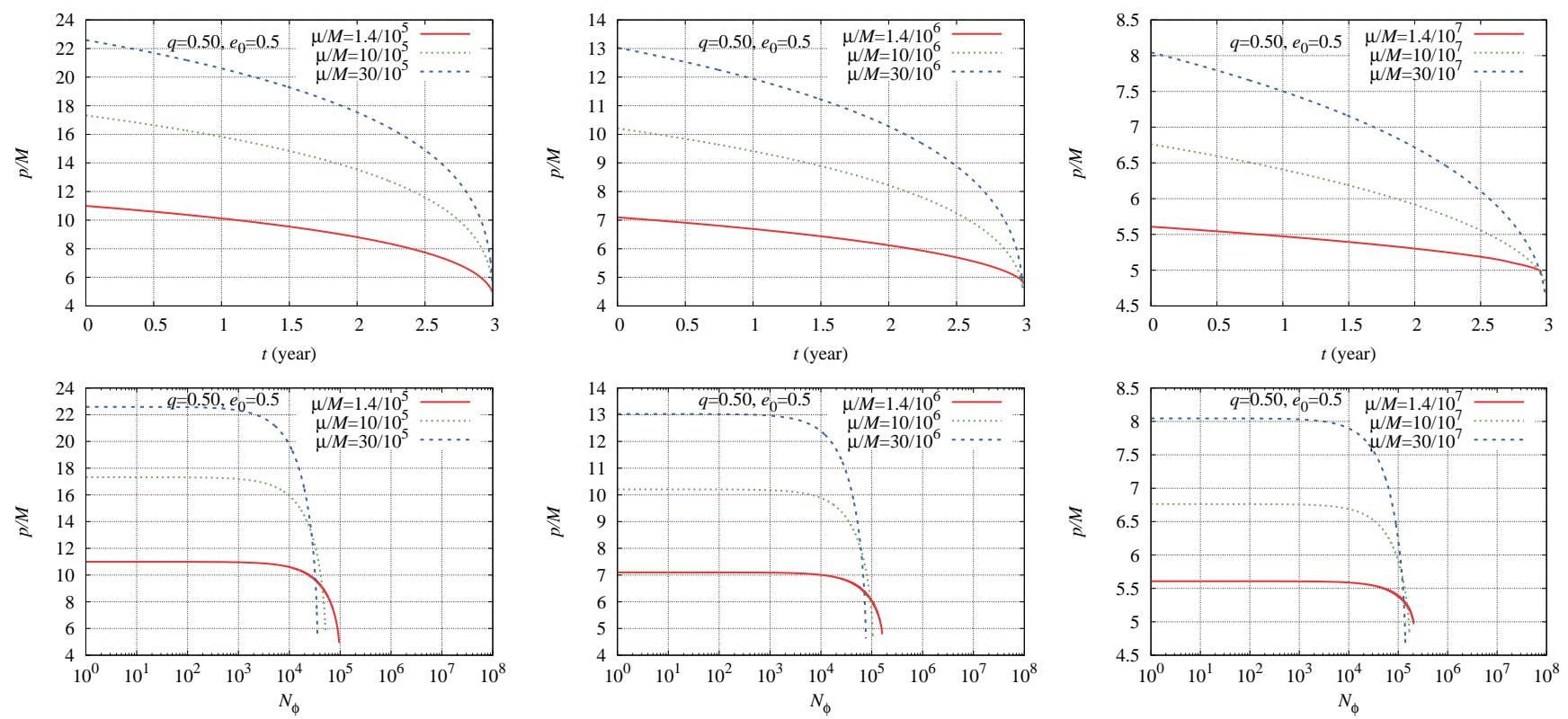

FIG. 1. Top panels show the evolution of the semilatus rectum $p$ as a function of time for the last 3-year inspirals before plunge with $q=0.5, M=10^{5} M_{\odot}$ (left), $10^{6} M_{\odot}$ (middle), and $10^{7} M_{\odot}$ (right), and the initial orbital eccentricity $e_{0}=0.5$. Bottom panels show $p$ as a function of orbital cycles for the last 3-year inspirals before plunge with the same parameters $\left(q, e_{0}, \mu, M\right)$ as in the top panels.

number of parameter sets of $(p, e)$ in reasonable computational time. Thus, we first compute them for the restricted data points and use an interpolation method to obtain $(\langle d p / d t\rangle,\langle d e / d t\rangle)$ at arbitrary points inside the region of the defined parameter space of $(p, e)$. Then, we obtain an inspiral orbit using the interpolated values of $(\langle d p / d t\rangle,\langle d e / d t\rangle)$. We employ a fifth-order Lagrange interpolation [43], both in $p$ and $e$, for this. We note that the similar method is employed, e.g., in Ref. [27] for the local fitting of gravitational self-force in Schwarzschild spacetime.

In this paper, we compute $(\langle d p / d t\rangle,\langle d e / d t\rangle)$ for $\approx$ 18000 data points in the $(p, e)$ space for each value of $q$. The number of the sampling points for $p$ is 256 for the range of $p_{\text {ISO }} \leq p \leq 30 M$ with a log-even spaced grid. Here, $p_{\text {ISO }}$ denotes $p$ at the innermost stable orbit for each value of $q$. For $e$, the sampling point is chosen to be $e=0.005,0.01$, and $0.0125 \leq e \leq 0.925$ with the grid spacing $\Delta e=0.0125$ (the total number is 76 ). The total computational cost for determining the gravitationalwave fluxes with these sampling points and $|q|=0,0.1$, $0.3,0.5,0.7$, and 0.9 is about $20 \mathrm{~d}$ using $\sim 400$ processors with $\sim 2.6 \mathrm{GHz}$ clock speed.

Figure 1 illustrates the results for inspiral orbits determined by the above procedure for $q=0.5$ and $e_{0}=0.5$ with $M=\left(10^{5}, 10^{6}, 10^{7} M_{\odot}\right)$ and $\mu=\left(1.4,10,30 M_{\odot}\right)$. Here, $e_{0}$ denotes the initial orbital eccentricity. We plot the evolution of $p$ and the number of orbital cycles $N_{\phi}$ for the last 3 years before plunge of a stellar-mass compact object into SMBHs. This figure shows that the lifetime of the EMRIs becomes 3 years for the cases that $p / M \approx 5-$
20, depending on the masses of the SMBH and compact star. It also shows that the typical total cycles of the orbit is $N_{\phi} \sim 10^{5}$ (i.e., the typical total phase of gravitational waves is $\sim 10^{6} \mathrm{rad}$ ) for the last 3-year inspiral orbits before plunge. Thus, if we require that the error in a gravitational-wave phase model is smaller than $1 \mathrm{rad}$, the error of the gravitational-wave fluxes has to be within $10^{-6}$.

\section{B. Accuracy of gravitational-wave fluxes}

The adiabatic change of the orbital parameters is computed from gravitational-wave fluxes, i.e., $\tilde{Z}_{\ell m n}^{\infty / \mathrm{H}}$, where we omit the $k$-mode because we focus on the equatorial orbits. For the computation of $\tilde{Z}_{\ell m n}^{\infty / \mathrm{H}}$, one needs to integrate ${ }_{-2} S_{\ell m}^{a \omega}(\theta)$ and $R_{\ell m \omega}(r)$ with the source term $T_{\ell m \omega}$ along a geodesic orbit. We use the numerical methods developed in Refs. [28, 29] to compute ${ }_{-2} S_{\ell m}^{a \omega}(\theta)$ and $R_{\ell m \omega}(r)$. One can compute them with the machine precision in most cases. However, in some cases, the accuracy of $R_{\ell m \omega}(r)$ is limited by that of the so-called renormalized angular momentum $\nu$ introduced in Refs. [30-32]: it is infeasible to accurately determine $\nu$ for large values of $M \omega$, typically $M \omega>3$ for $(\ell, m)=(2,2)$ in double precision calculation [28, 29] (see, however, Refs. [44-47] which use Mathematica codes to determine $\nu$ in high precision, $\sim 100$ decimal places). Since the high-frequency modes play an important role, it becomes challenging to accurately compute gravitational-wave fluxes for compact orbits (with small values of $r_{\min }$ ), in particular for 
the high $\mathrm{BH}$ spin of $q \gtrsim 0.9$.

We use the trapezium rule, which has an excellent convergence property to integrate periodic functions, to derive $\tilde{Z}_{\ell m n}^{\infty / \mathrm{H}}[23,47]$. We choose the maximum number of the grid points in the trapezium rule as $2^{14}+1$ to save computational time. In Ref. [23], with this number of the grid points, it is found that one can compute $d I^{i} / d t$ with the accuracy of $10^{-10}$ for $q=0, p=10 M$, and $e=0.9$. However, the numerical accuracy is not as good as this level for a high value of $q \gtrsim 0.9$ as we find in the present work. To summarize, the numerical accuracy in $\tilde{Z}_{\ell m n}^{\infty / \mathrm{H}}$ is currently limited by that of $\nu$ and the number of the grid points used in the trapezium rule for a high value of $q \gtrsim 0.9$. Improving the accuracy for this special case is the issue left for the future work.

The numerical accuracy in the gravitational-wave fluxes is also limited by truncating the mode summation in Eqs. (35) and (36). In the present work, the mode summation in the fluxes, Eqs. (35) and (36), is performed until the error becomes smaller than $10^{-6}$ at least for $p \geq 6 M$ and $e \leq 0.8$. We choose this error size because the total cycle of gravitational waves during a few years LISA observation is of the order of $10^{5}$ as already illustrated in Fig. 1.

The mode summation in Eqs. (35) and (36) is expressed as

$$
\begin{gathered}
F=\sum_{\ell=2}^{\infty} F_{\ell} \\
F_{\ell}=\sum_{m=-\ell}^{\ell} F_{\ell m}, \\
F_{\ell m}=2 \sum_{n=n_{i}}^{\infty} F_{\ell m n},
\end{gathered}
$$

where $n_{i}$ is the minimum integer which satisfies $m \Upsilon_{\phi}+$ $n_{i} \Upsilon_{r}>0$, and

$$
F=\left\langle\frac{d \mathcal{E}}{d t}\right\rangle \quad \text { or } \quad\left\langle\frac{d \mathcal{L}_{z}}{d t}\right\rangle .
$$

In Eq. (43), we used the relation of $F_{\ell m n}=F_{\ell-m-n}$ to take into account the modes of $M \omega<0$.

We truncate the $\ell$-summation in Eq. (41) by choosing a maximum value of $\ell$ as $\ell_{\max }$. Then, $F$ is written as

$$
F=\sum_{\ell=2}^{\ell_{\max }} F_{\ell}+\delta F_{\ell_{\max }}
$$

where $\delta F_{\ell_{\max }}$ is the error due to restricting the $\ell$ summation up to $\ell=\ell_{\max }$. The reason that we set the maximum value of $\ell$ is that for very high values of $\ell \gtrsim \ell_{\max }$, the value of $\nu$ cannot be numerically calculated accurately. As mentioned above, this problem could be fixed if we can improve the precision for the numerical calculation of $\nu$.

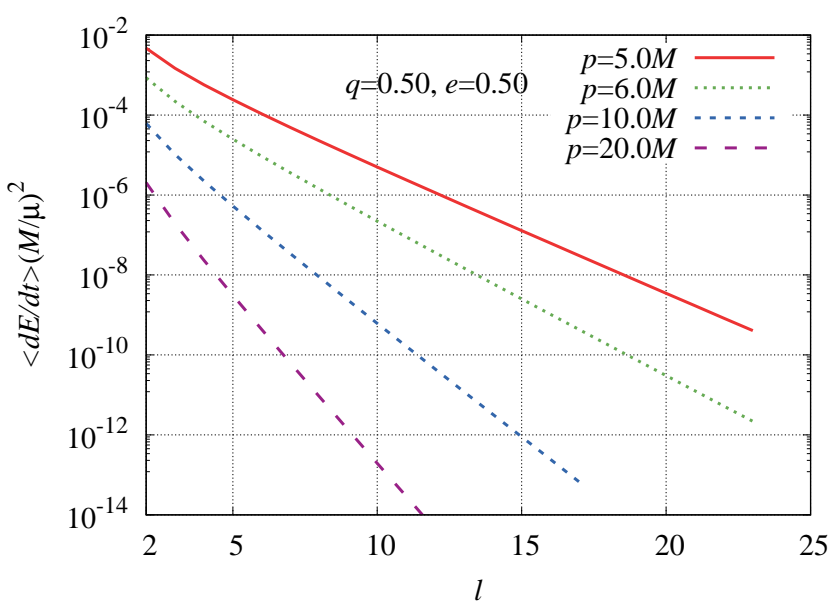

FIG. 2. Gravitational-wave energy flux $\left(F_{\ell}\right)$ as a function of $\ell$ for $q=0.5, e=0.5$, and $p=5.0 M, 6.0 M, 10.0 M$, and $20.0 M$.

Figure 2 shows the energy flux of gravitational waves, $F_{\ell}$, as a function of $\ell$ for $q=0.5$ and $e=0.5$. $F_{\ell}$ decreases approximately exponentially with the increase of $\ell$, but the decrease rate becomes less steep for smaller values of $p$. If we assume the exponential decrease of $F_{\ell}$ in $\ell$, the error size by the truncation of the higher- $\ell$ modes, $\delta F_{\ell_{\max }}$, can be estimated as

$$
\delta F_{\ell_{\max }}=F \sum_{\ell=\ell_{\max }+1}^{\infty} \mathrm{e}^{-\alpha \ell}=F \frac{\mathrm{e}^{-\alpha \ell_{\max }}}{\mathrm{e}^{\alpha}-1}
$$

where $\alpha$ is a positive constant.

In this paper the maximum value of $\ell$ is set to be $\ell_{\max }=25$. This implies that $\delta F_{\ell_{\max }} / F$ is less than $10^{-6}$ for $\alpha \gtrsim 0.6$. Figure 3 shows $\alpha$ as a function of $r_{\min }$ for $q=-0.5$ (left), 0 (middle), and 0.5 (right) with several values of $e$. We find that the value of $\alpha$ is larger than 0.6 for any stable orbits with $q \lesssim 0.5$ for which the minimum value of the orbital radius, $r_{\text {min }}$, is larger than $\sim 3 M$. Hence, we conclude that the error due to restricting the $\ell$-summation up to $\ell_{\max }=25$ in the energy dissipation rate for $q \lesssim 0.5$ is less than $10^{-6}$. However, $\ell_{\max }=25$ is not large enough to achieve the required error size for orbits close to the separatrix with $q \gtrsim 0.6$.

The summation over the $n$-modes in Eq. (43) is approximated as

$$
F_{\ell m}=2 \sum_{n=n_{i}}^{n_{f}} F_{\ell m n}
$$

where $n_{f}$ is determined by $F_{\ell m n_{f}}<10^{-6} F$. We note that the values of $n$ for the dominant modes of $F_{\ell m n}$ shifts to larger values of $n$ for larger values of $\ell$ and $e$ (see Refs. [21, 23]), and $F_{\ell m n}$ decreases exponentially with the increase of $n$ after the dominant mode of $F_{\ell m n}$ is reached [21, 23]. In this paper, the maximum value of $n_{f}$ is set to be 1000 . This choice is large enough for the orbits with $e \lesssim 0.8$. 

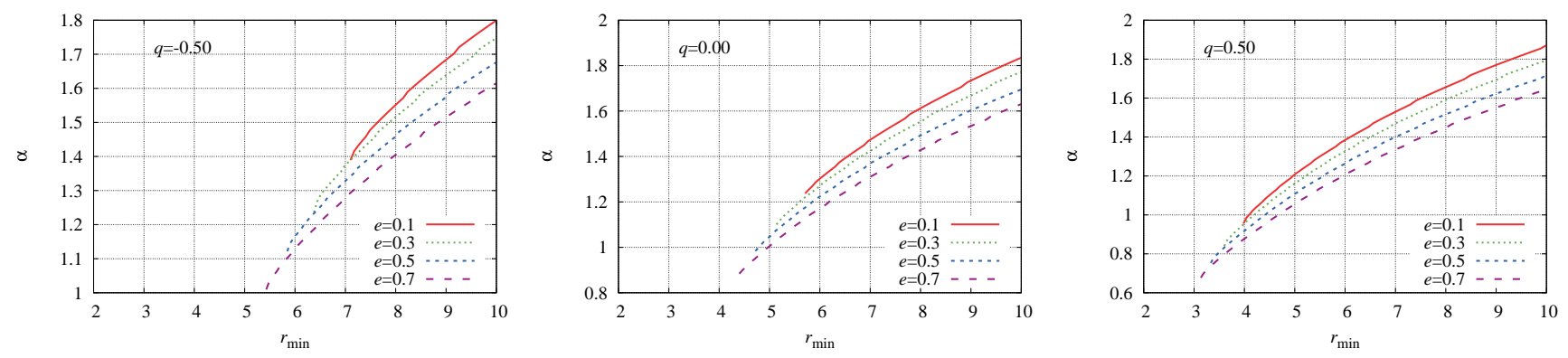

FIG. 3. $\alpha$ as a function of $r_{\min }$ for $q=-0.5$ (left), 0 (middle), and 0.5 (right).

Figure 4 shows the energy spectrum during the 3-year inspiral before plunge for $q=0.5$ and $e_{0}=0.6$ with $M=10^{6} M_{\odot}$ and $\mu=10 M_{\odot}$. The values of $(p, e)$ take $(10.1 M, 0.60)$ at the beginning, $(8.9 M, 0.50)$ at 1.5 years, and $(4.6 M, 0.24)$ at the plunge, respectively. We note that the number of the $n$-modes necessary for the required accuracy for fixed values of $(\ell, m)$ decreases as approaching the separatrix because of the circularization of the orbital eccentricity (see, e.g., Figs. 6 and 7). By contrast, the number of the $\ell$-modes necessary for the required accuracy increases with the orbital evolution, because the value of $r_{\min }$ decreases and relativistic effects are enhanced with the orbital evolution. To achieve the relative error in the energy dissipation rate within $10^{-6}$, the maximum values of $(\ell, n)$ become $(18,109),(19,76)$, and $(24,42)$ at the beginning, 1.5 years, and the plunge, respectively.

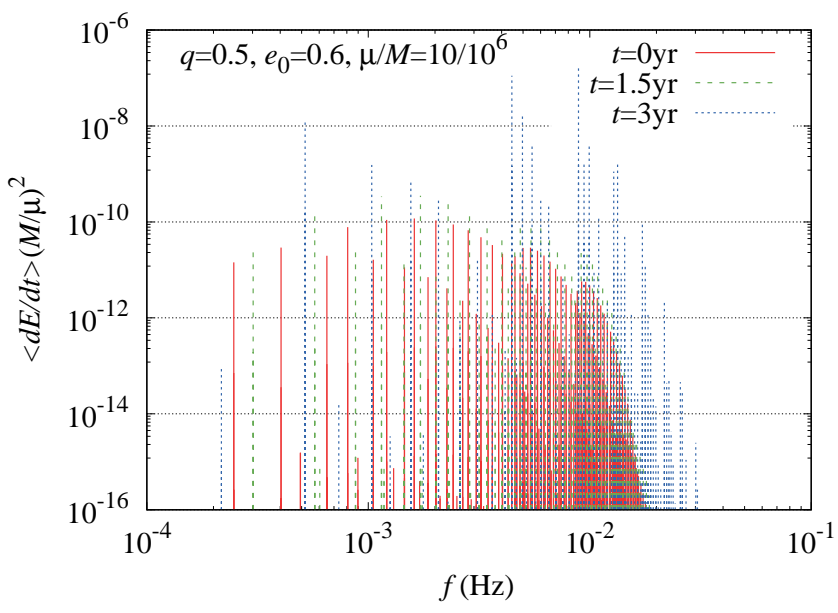

FIG. 4. The energy spectrum during the 3-year inspiral before plunge for $q=0.5$ and $e_{0}=0.6$ with $M=10^{6} M_{\odot}$ and $\mu=10 M_{\odot}$. The values of $(p, e)$ take $(10.1 M, 0.60)$ at $t=0$ year, $(8.9 M, 0.50)$ at $t=1.5$ years, and $(4.6 M, 0.24)$ at $t=3$ years, respectively.

To confirm the validity of the interpolation, the relative error in the interpolated energy flux is estimated by comparison with numerical data independent of those used for the interpolation, and the results are shown in
Fig. 5. This shows that the error is smaller than $10^{-6}$ for $r_{\min }=p /(1+e) \gtrsim 3 M$. Thus, the required accuracy is always achieved for $q \leq 0.5$. As already mentioned, the accuracy is also limited by the accuracy of $\nu$, the number of the grid points used in the trapezium rule, and the truncation with respect to the $\ell$-summation. By these limitations, the accuracy with the error less than $10^{-6}$ is not achieved for the compact orbits of $r_{\min } \lesssim 3 M$. Figure 5 shows the similar feature for the magnitude of the error associated with the interpolation. This suggests that the accuracy would be limited by that for the individual data set, not by the interpolation. To conclude, currently, for the case that the value of $r_{\min }$ is smaller than $3 M$ (i.e., for $q \gtrsim 0.6$ ), the accuracy of $10^{-6}$ is not achieved due to the error of the individual data set.

Here, we should note the following point: the lifetime of the binaries with an orbit near the separatrix to plunge is so short that the total cycle of the orbits is at most $10^{4}$ (see Fig. 1). This indicates that for such compact orbits, the accuracy of $\lesssim 10^{-5}$ would be acceptable. Thus in this paper, we believe that the accuracy of our numerical results for the inspiral orbits is acceptable for $q \leq 0.7$. However, for $q \geq 0.9$, we should keep in mind that the accuracy is not sufficient. Improving the accuracy for the case of $q$ close to unity is the issue to be solved in the future work.

\section{RESULTS}

In this section, we present inspiral orbits and corresponding gravitational-wave spectra for the typical EMRIs as sources of LISA using the procedure described in Sec. III. We also calculate the SNR of gravitational waves for such EMRIs using the LISA's designed sensitivity curve.

\section{A. Inspiral orbits}

Figures 6 and 7 illustrate the inspiral orbits showing the orbital eccentricity $e$ as a function of the semilatus rectum $p$ for several values of $\left(q, p_{0}, e_{0}\right)$. Here, $p_{0}$ and $e_{0}$ are the initial semilatus rectum and the initial orbital ec- 

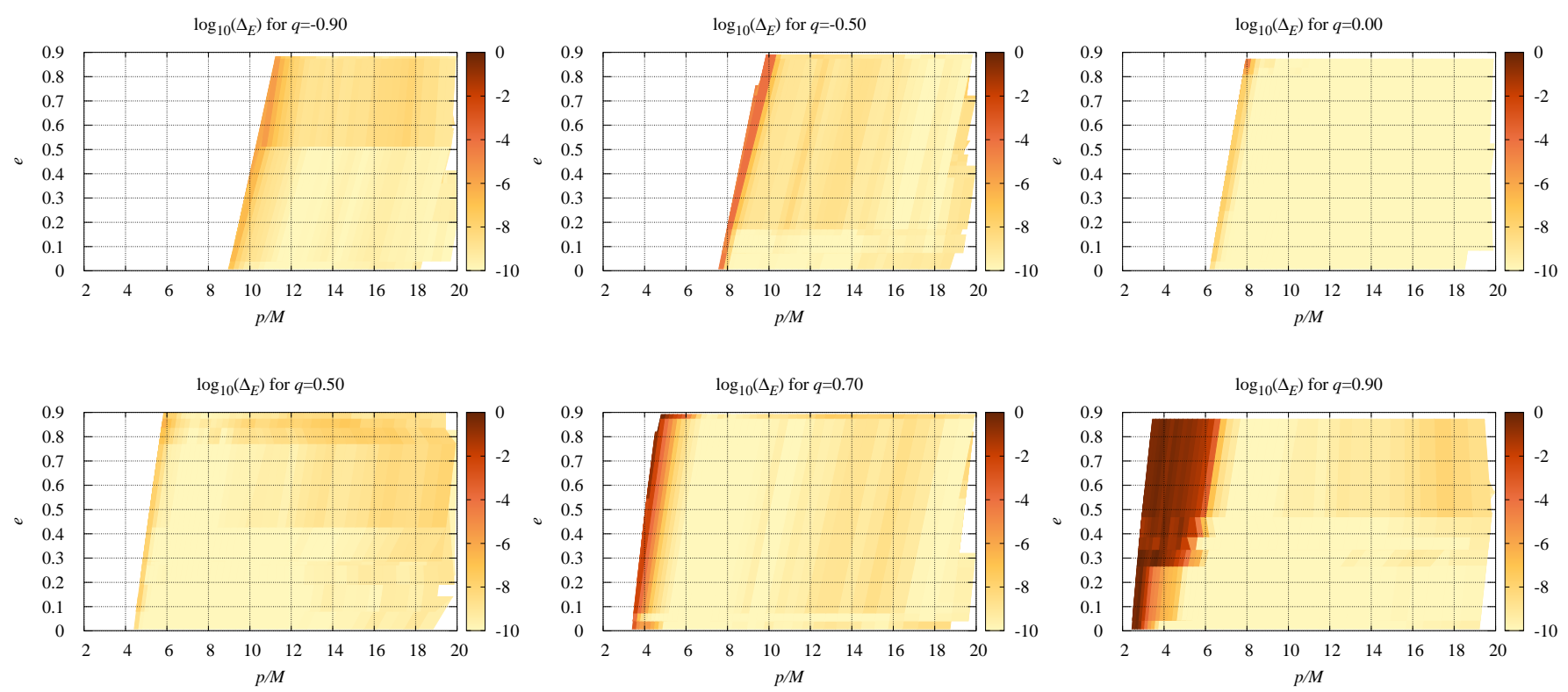

FIG. 5. Relative error in the interpolated energy flux, $\Delta_{\mathrm{E}}$, for $q=-0.9,-0.5,0.0,0.5,0.7$, and 0.9 . The error is estimated by comparison with numerical data independent of those used for the interpolation. The error is smaller than $10^{-6}$ for $r_{\min }=p /(1+e) \gtrsim 3 M$.
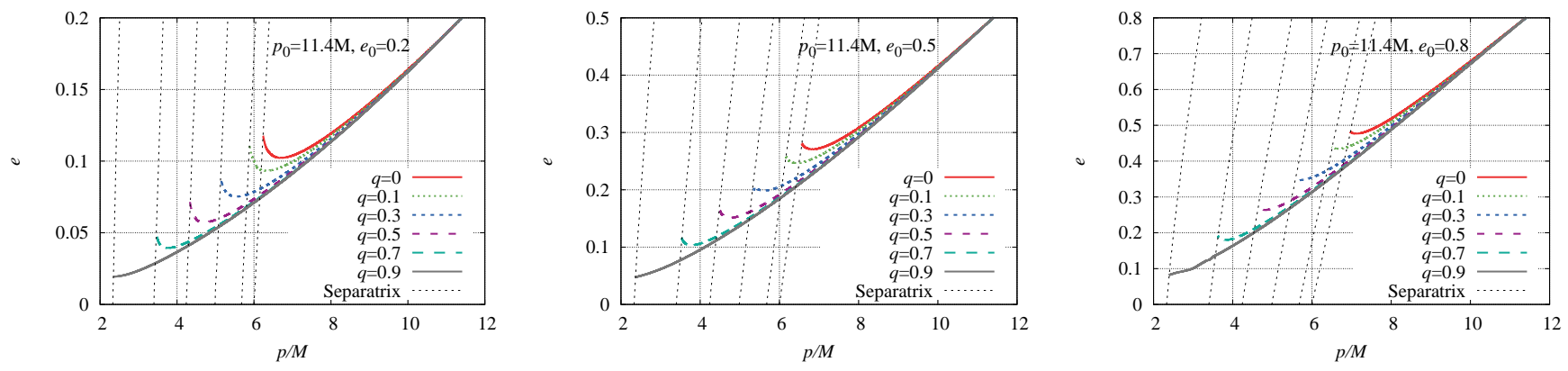

FIG. 6. The orbital eccentricity $e$ as a function of the semilatus rectum $p$ for inspiral orbits from $p_{0}=11.4 M$ to the separatrix with $q=0,0.1,0.3,0.5,0.7$, and 0.9 . The initial orbital eccentricity is chosen to be $e_{0}=0.2$ (left), 0.5 (middle), and 0.8 (right). The dashed curves show the separatrix of stable orbits. Note that it takes $\sim 100 M^{2} / \mu$ for $q=-0.5, \sim 190 M^{2} / \mu$ for $q=0$ and $\sim 300 M^{2} / \mu$ until plunge for $q=0.5$ with $e_{0}=0.2$ (see also Fig. 17). The inspiral time becomes longer for the larger $\mathrm{BH}$ spin with fixed values of $p_{0}$ and $e_{0}$ because $p$ at the separatrix becomes smaller. We note that the orbits near the separatrix are not obtained for $q=0.9$ as accurately as for other values of $q$ (see a discussion in Sec. III).
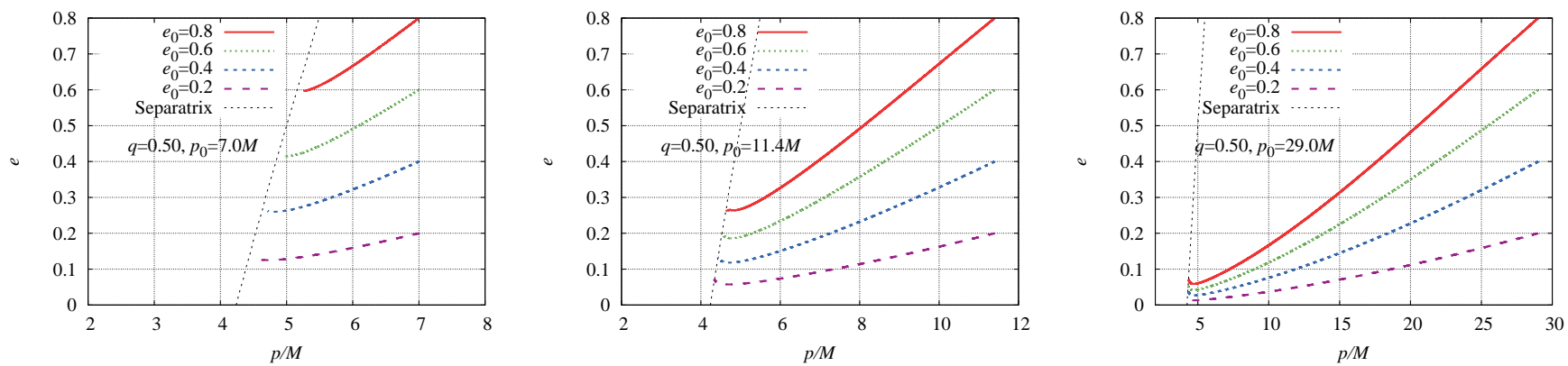

FIG. 7. The same as Fig. 6, but for inspiral orbits from $p_{0}=7.0 M$ (left), $11.4 M$ (middle), and $29.0 M$ (right) to the separatrix for $q=0.5$ with $e_{0}=0.8,0.6,0.4$, and 0.2 . The dashed curves denote the separatrix of stable orbits for $q=0.5$. 
centricity, respectively. In Fig. 6, the inspirals start from $p_{0}=11.4 M$ to the separatrix with $e_{0}=0.2$ (left), 0.5 (middle), and 0.8 (right) for $q=0$ to 0.9 . We note that in our assumption imposed in this paper, the curves $e(p)$ do not depend on $M$ and $\mu$ [48]. It takes $\sim 100 M^{2} / \mu$ for $q=-0.5, \sim 190 M^{2} / \mu$ for $q=0$, and $\sim 300 M^{2} / \mu$ for $q=0.5$ from $p_{0}=11.4 M$ to the plunge with $e_{0}=0.2$ (cf. also Fig. 17). Here, $\sim 300 M^{2} / \mu$ is $\sim 5$ years for $M=10^{6} M_{\odot}$ and $\mu=10 M_{\odot}$. We note that the inspiral time becomes longer for the larger $\mathrm{BH}$ spin with fixed values of $p_{0}$ and $e_{0}$ because $p$ at the separatrix becomes smaller. The inspiral time also becomes longer for the larger values of $e_{0}$ with a fixed value of $p_{0}$, because the adiabatic change of $p$ (i.e., $\langle d p / d t\rangle$ ) becomes smaller for larger values of $e$ with a fixed value of $p$ [48]. Figure 6 shows that the circularization of the orbital eccentricity occurs for the most stages of the inspiral, but the eccentricity slightly increases near the separatrix as already found in Refs. [19, 20, 23, 26, 27].

Figure 7 compares the inspiral orbits with $p_{0}=7.0 \mathrm{M}$ (left), 11.4M (middle), and 29.0M (right), fixing $q=0.5$, but varying $e_{0}$ from 0.8 to 0.2 . This also shows that the orbits always circularize in the early inspiral, but the eccentricity increases as approaching the separatrix. For the larger value of $p_{0}$, the plunge occurs for a small value of $p$, which is approximately equal to the radius of the innermost stable circular orbit. Thus, for the case that $p_{0} / M$ is fairly large $\sim 30$, the circularization occurs significantly even for $e_{0}=0.8$ and results in the nearly circular orbits just before the plunge. For relatively small SMBH mass, e.g., $M \sim 10^{5} M_{\odot}$, the inspiral proceeds from $p_{0} \approx 30 M$ to the plunge in a few years for $\mu \sim$ $10 M_{\odot}$. For such a case, the final orbit is likely to be nearly circular even if $e_{0}$ is initially high as $e_{0}=0.8$. By contrast, for a higher value of $M$, the inspiral time of stellar-mass object is a few years even if $p_{0}$ is smaller than $10 M$. For the small value of $p_{0}$, the eccentricity does not change significantly until the plunge orbit is reached. Thus such a plunge orbit could have a large eccentricity if $e_{0}$ at $p<10 M$ is so. We note that the two-body relaxation in star clusters of the galactic centers could produce highly eccentric EMRIs with $p \leq 10 M$ in the LISA band that do not plunge immediately because the value of $p$ at the separatrix becomes smaller than that of a Schwarzschild $\mathrm{BH}$ if one takes into account the $\mathrm{BH}$ spin [49].

\section{B. Gravitational-wave spectra and SNR}

Following Ref. [50], the squared SNR averaged over all source directions is defined by

$$
\mathrm{SNR}^{2}=4 \int_{0}^{\infty} d(\log f)\left[\frac{h_{\mathrm{eff}}(f)}{h_{\mathrm{n}}(f)}\right]^{2},
$$

where $h_{\mathrm{n}}(f)$ is the noise amplitude and $h_{\mathrm{eff}}(f)$ is the power spectrum defined below. The noise amplitude is defined by $h_{\mathrm{n}}(f)=\sqrt{f S_{\mathrm{n}}(f)}[51]$, where $S_{\mathrm{n}}(f)$ is the onesided noise power spectral density. In this paper, $S_{\mathrm{n}}(f)$ is taken to be the analytic form of the LISA's designed sky-averaged sensitivity presented in Ref. [4].

The power spectrum is defined by the summation of the power spectrum for $\ell, h_{\text {eff }}^{\ell}$, as

$$
h_{\mathrm{eff}}(f)=\sum_{\ell} h_{\mathrm{eff}}^{\ell}(f)
$$

where

$$
h_{\mathrm{eff}}^{\ell}(f)=\sum_{m n} h_{\mathrm{eff}}^{\ell m n}(f),
$$

and $h_{\mathrm{eff}}^{\ell m n}(f)$ is estimated by [50]

$$
h_{\mathrm{eff}}^{\ell m n}\left(f_{\ell m n}\right)=\frac{1}{\pi D} \sqrt{\frac{2 \dot{E}_{\ell m n}}{\dot{f}_{\ell m n}}} .
$$

Here, the dot denotes the time derivative, $D$ is the distance to the source, $\dot{E}_{\ell m n}$ is the energy flux to infinity due to the emission of gravitational waves at frequency $f_{\ell m n}$, which is defined as

$$
f_{\ell m n}=\frac{m \Upsilon_{\phi}+n \Upsilon_{r}}{2 \pi \Gamma} \equiv m f_{\phi}+n f_{r}
$$

where Eq. (33) is used. $f_{\phi}$ and $f_{r}$ are the frequencies of the azimuthal and radial motion, respectively. In our inspiral orbits, $f_{\phi}$ and $f_{r}$ can be computed at each time step by using $p(t)$ and $e(t)$. We then compute $\dot{f}_{\ell m n}$ from $\Delta f_{\ell m n} / \Delta t$, where $\Delta t$ is the time step for evolving the orbital motion and $\Delta f_{\ell m n}=f_{\ell m n}(t+\Delta t)-f_{\ell m n}(t)$ (see Sec. III A for our choice of the time step). We use $\approx$ 100 frequency bins to smooth the modal power spectrum $h_{\mathrm{eff}}^{\ell m n}(f)$. We compute $h_{\mathrm{eff}}^{\ell m n}(f)$ for the modes of $\ell=2-4$, $-\ell \leq m \leq l$, and $n_{0} \leq n \leq n_{0}+45$ where $n_{0}=-m$. We choose this value of $n_{0}$ in order to compute the SNR with the relative error of $\lesssim 10 \%$. We check the error by varying $n_{0}$ from 10 to 60 . For $e_{0}=0.4$ and $\ell=2, n_{0}=$ 10 is sufficient to compute the SNR with such accuracy. However, we need a larger value of $n_{0}$ for higher values of $e_{0}$ and $\ell$ [21, 23]. For $e_{0}=0.8$ and $\ell=2(\ell=4)$, we need $n_{0}=30\left(n_{0}=45\right)$ to compute the SNR with the error of $\lesssim 10 \%$. The power spectrum $h_{\text {eff }}(f)$ is computed by summing all the modes of $h_{\mathrm{eff}}^{\ell m n}(f)$ at each frequency bin.

In Fig. 8, we compare the power spectrum for $\ell=2$ derived from our numerical results with those obtained by kludge models [52-55] as a consistency check. The power spectra for the kludge models are obtained in the following manner. First, we compute the time domain gravitational waveforms using the EMRI Kludge Suite in the Black Hole Perturbation Toolkit [56]. The public code enables us to compute the inspiral orbits, the time domain waveforms, and the SNR for given parameters such as $\left(q, p_{0}, e_{0}, \mu, M, D, T, \Delta t\right)$, where $T$ is the duration of the waveform and $\Delta t$ is a time step. The waveforms 


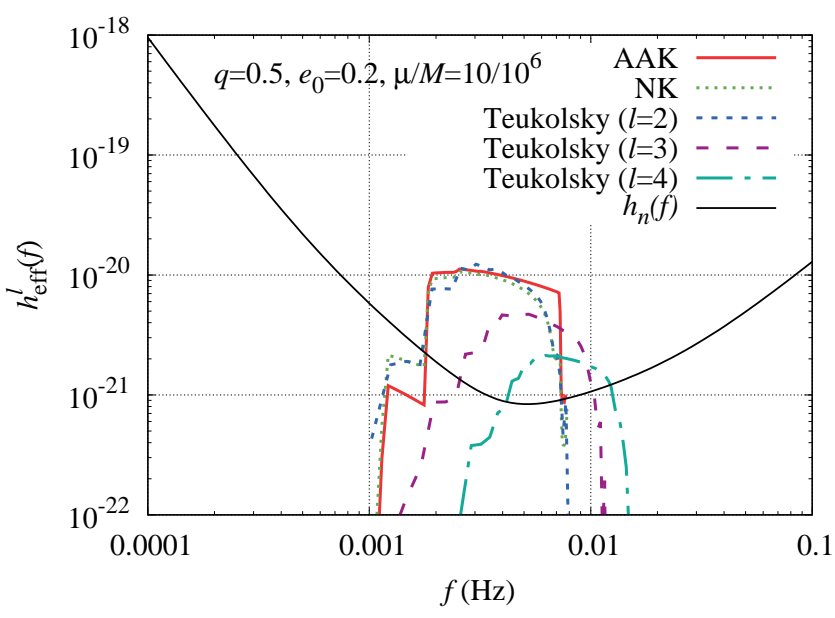

FIG. 8. Power spectra with $\ell=2$ for numerical kludge (NK) [52, 53], augmented analytic kludge (AAK) [54], and Teukolsky (this work) models for a $10 M_{\odot}$ compact object inspiraling around a $10^{6} M_{\odot} \mathrm{SMBH}$ of spin $q=0.5$ at $D=1 \mathrm{Gpc}$ during the last 3-year inspiral before plunge. We consider the inspiral with $p_{0}=10.3 M$ and $e_{0}=0.2$. The amplitudes in the Teukolsky model for $\ell=3$ and 4 modes are also shown. The curve $h_{\mathrm{n}}(f)$ shows the LISA's designed sky-averaged sensitivity [4].

are given by LISA response functions $h_{I}$ and $h_{I I}$, which are transformed from the waveform polarizations $h_{+}$and $h_{\times}$as

$$
\begin{aligned}
h_{I} & =\frac{\sqrt{3}}{2}\left(F_{I}^{+} h_{+}+F_{I}^{\times} h_{\times}\right), \\
h_{I I} & =\frac{\sqrt{3}}{2}\left(F_{I I}^{+} h_{+}+F_{I I}^{\times} h_{\times}\right),
\end{aligned}
$$

where $F_{I}$ and $F_{I I}$ are the antenna pattern functions [57]. We choose $\Delta t=63 \mathrm{~s}$ to compute the inspiral orbits and the time domain waveforms, which are constructed from $\sqrt{h_{I}^{2}+h_{I I}^{2}}$. We then perform Fourier transformation for the time domain waveforms of the kludge models into the frequency domain, and smooth $h_{\mathrm{eff}}(f)$ by using 100 frequency bins.

The power spectra are computed for a $10 M_{\odot}$ compact object inspiraling around a $10^{6} M_{\odot} \mathrm{SMBH}$ of spin $q=0.5$ at $D=1 \mathrm{Gpc}$ during the last 3 -year inspiral before plunge. For this setting, the inspiral starts from $p_{0}=10.3 M$ with $e_{0}=0.2$. We compute the power spectra $h_{\mathrm{eff}}(f)$ for the numerical kludge $(\mathrm{NK})$ model $[52,53]$ and the augmented analytic kludge (AAK) model [54]. Here, in the NK, the orbital motion is determined by solving the geodesic equation with the gravitational radiation reaction based on post-Newtonian (PN) formulas [58], which are fitted to the $\mathrm{BH}$ perturbation theory. In the AAK, the orbital motion is determined by solving the geodesic equation with the gravitational radiation reaction based on $\mathrm{PN}$ formulas in the $\mathrm{BH}$ perturbation theory [59]. Gravitational waveforms are determined from the resulting orbits using the quadrupole formula [60] both in the NK and AAK models.

Figure 8 shows that the power spectrum for the $\ell=2$ mode derived in our calculation is closer to the one by the NK model than that by the AAK model. This is consistent because the NK model should be more accurate than the AAK model. In Fig. 8, the LISA's designed sky-averaged sensitivity written in an analytic form [4] is also shown. It is found that for EMRIs with $\mu=10 M_{\odot}$ together with an SMBH of $M=10^{6} M_{\odot}$ and of $q=0.5$ at $D=1 \mathrm{Gpc}$, the SNR is of the order of 10 (more details on the SNR will be presented below).

The power spectra for $\ell=3$ and 4 modes derived in our calculation are also shown in Fig. 8. This illustrates that the modes with $\ell=3$ and 4 have the amplitude approximately by $40 \%$ and $20 \%$ as large as that for the $\ell=2$ mode, respectively (see also Fig. 13 for the SNR associated with the $\ell=3$ and $\ell=4$ modes). This is reasonable because the orbit which we consider here is very general relativistic, and hence, the orbital velocity can be $\approx 50 \%$ of the speed of light, resulting in the enhancement of the higher multipole modes. We note that the amplitudes for the $\ell \geq 5$ modes are less than $10 \%$ of that for the $\ell=2$ mode. We ignore the amplitudes for the $\ell \geq 5$ modes because they are smaller than $h_{\mathrm{n}}(f)$ for $e_{0} \lesssim 0.8$.

To explore the dependence of the spectrum feature on the initial orbital eccentricity and the $\mathrm{BH}$ spin, we generate Figs. 9 and 10. These figures show the spectra of gravitational waves emitted by a $10 M_{\odot}$ compact object inspiraling around a $10^{6} M_{\odot} \mathrm{SMBH}$ at $D=1 \mathrm{Gpc}$ for the last 3-year inspiral before plunge for a variety of $q$ and $e_{0}$. In Fig. 9, the BH spin is fixed to be $q=0.5$, while $e_{0}$ is varied from 0.2 to 0.8 . The values of $p_{0}$ take $10.33 M$, $10.28 M, 10.11 M$, and $9.58 M$ for $e_{0}=0.2,0.4,0.6$, and 0.8 , respectively. As the initial eccentricity increases, the maximum frequency of gravitational waves becomes higher, because with the large eccentricity, the minimum value of $r_{\min }$ is smaller resulting in the excitation of the higher frequency modes (see Fig. 7 and Ref. [55]): e.g., for $\ell=m=2$, the $n=0$ mode is dominant for $e_{0} \lesssim 0.1$, while the $n=0$ and 1 modes are equally dominant for $e_{0} \approx 0.3$ and the $n=2$ and 3 modes become dominant for $e_{0} \approx 0.7$.

We find that the maximum value of $h_{\mathrm{eff}}(f)$ increases as the value of $e_{0}$ increases (see also Fig. 13 for the SNR as a function of $\left.e_{0}\right)$. The reason for this is that $r_{\min }$ decreases with the increase of $e_{0}$, resulting possibly in the enhancement of the gravitational-wave amplitude. Moreover, we need to sum over larger number of $n$-modes as the value of $e_{0}$ increases, and hence, the maximum value of $h_{\mathrm{eff}}(f)$ at a peak frequency increases. Indeed, the power spectrum becomes broader in frequency as the value of $e_{0}$ increases.

In Fig. 10, the initial orbital eccentricity is fixed to be $e_{0}=0.4$, while the $\mathrm{BH}$ spin is varied from $q=0$ to 0.9 . The values of $p_{0}$ take $11.38 M, 11.15 M, 10.71 M, 10.28 M$, $9.83 M$, and $9.41 M$ for $q=0,0.1,0.3,0.5,0.7$, and 0.9 , respectively. The frequency of gravitational waves 

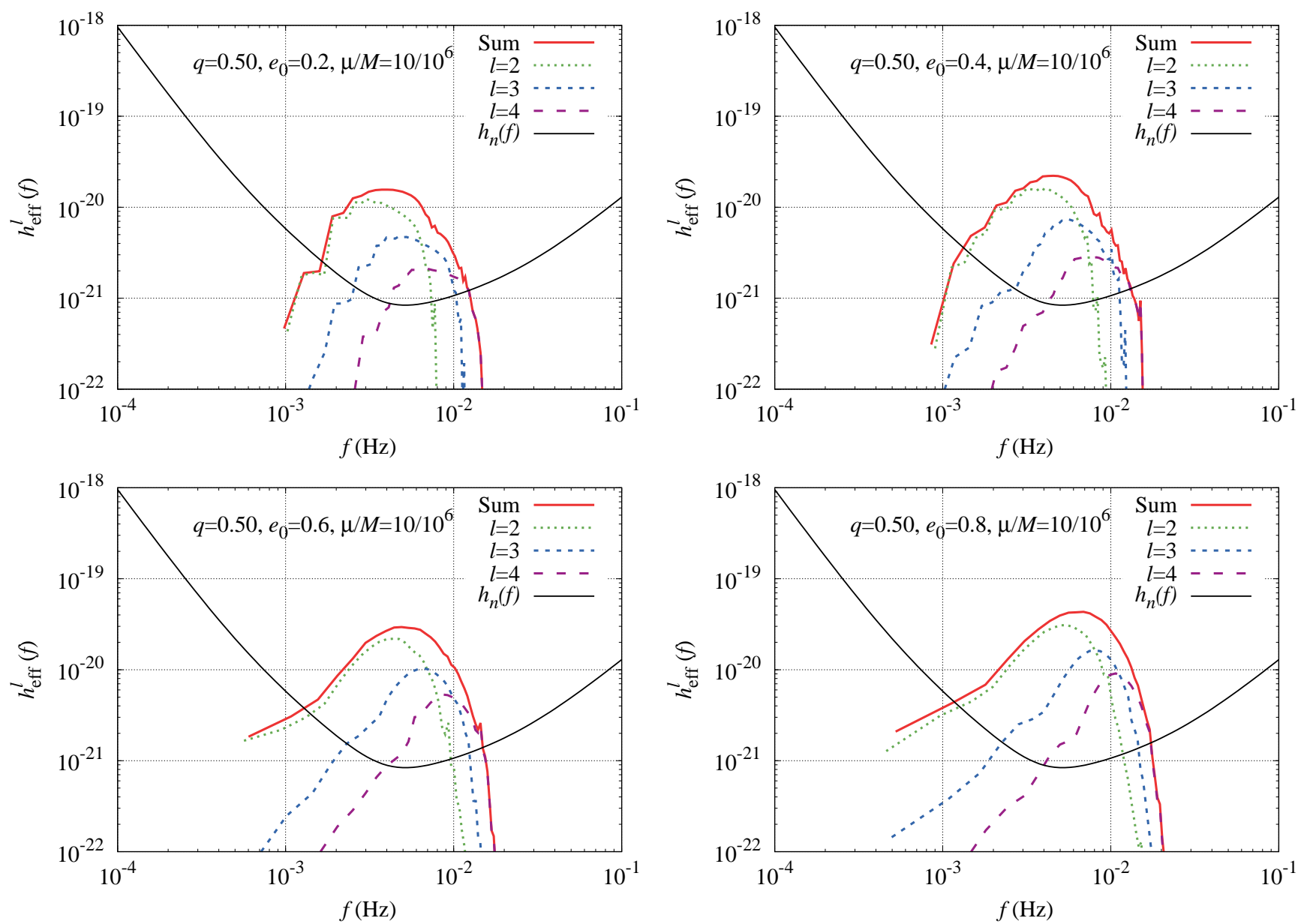

FIG. 9. Power spectra for $\ell=2,3$, and 4 induced by a $10 M_{\odot}$ compact object inspiraling around a $10^{6} M_{\odot} \mathrm{SMBH}$ of spin $q=0.5$ at $D=1 \mathrm{Gpc}$ for the last 3-year inspiral before plunge. The initial orbital eccentricity is chosen to be $e_{0}=0.2$ (top left), 0.4 (top right), 0.6 (bottom left), and 0.8 (bottom right). The values of $p_{0}$ take $10.33 M, 10.28 M, 10.11 M$, and $9.58 M$ for $e_{0}=0.2$, 0.4, 0.6, and 0.8, respectively. Complicated structures in the amplitude can be understood by noting contributions from higher radial modes ( $n$-modes) to gravitational waves (see, e.g., Ref. [55]). The curve $h_{\mathrm{n}}(f)$ shows LISA's designed sky-averaged sensitivity [4].

at plunge becomes higher as the $\mathrm{BH}$ spin increases because the minimum value of $r_{\min }$ becomes smaller (see Fig. 6). We also note that the maximum value of the power spectrum becomes larger for the larger $\mathrm{BH}$ spin (see Fig. 13 for the SNR as a function of $q$ ). The reason for this is that for the larger $\mathrm{BH}$ spin, the more compact orbits with smaller values of $r_{\min }$ is allowed, and for such orbits, gravitational waves of the high amplitude can be emitted due to the more relativistic motion.

Figure 11 shows the power spectra for a compact object of mass $\mu=(1.4,10,30) M_{\odot}$ inspiraling around a SMBH of mass $10^{6} M_{\odot}$ at $D=1 \mathrm{Gpc}$ during the last 3year inspiral with $e_{0}=0.6$. The $\mathrm{BH}$ spin is varied from $q=-0.7$ to 0.7 . For $q=0.7(-0.7)$, the values of $p_{0}$ take $6.3 M(10.8 M), 9.6 M(12.9 M)$, and $12.5 M(15.2 M)$ for $\mu=1.4 M_{\odot}, 10 M_{\odot}$, and $30 M_{\odot}$, respectively. Both the frequency of gravitational waves and the power spectra become higher as the BH spin increases. The power spectra increase as $\mu$ increases, and the maximum amplitudes are approximately proportional to $\sqrt{\mu}$.

In Fig. 12, the spectra are shown for a $10 M_{\odot}$ compact object inspiraling around an $\mathrm{SMBH}$ of mass $\left(10^{5}, 10^{6}, 10^{7}\right) M_{\odot}$ at $D=1 \mathrm{Gpc}$ during the last 3 -year inspiral with $e_{0}=0.6$. The $\mathrm{BH}$ spin is again varied from $q=-0.7$ to 0.7 . For $q=0.7(-0.7)$, the values of $p_{0}$ are $16.8 M(18.9 M), 9.6 M(12.9 M)$, and $6.0 M(10.6 M)$ for $M=10^{5} M_{\odot}, 10^{6} M_{\odot}$, and $10^{7} M_{\odot}$, respectively. The maximum amplitude of the spectra increases as the $\mathrm{BH}$ mass increases because $p_{0} / M$ becomes smaller and thus the inspiral orbits are in more highly general relativistic regions for a longer duration (see Fig. 1). The frequency of gravitational waves at plunge becomes higher as the $\mathrm{BH}$ mass decreases and the $\mathrm{BH}$ spin increases. As a result, gravitational waves from a compact object around a $10^{7} M_{\odot}$ SMBH with $q<0$ are not well in the LISA sensitivity band. In addition, only gravitational waves in an early part of the inspiral of a compact object into a $10^{5} M_{\odot} \mathrm{SMBH}$ is above the LISA sensitivity curve. 

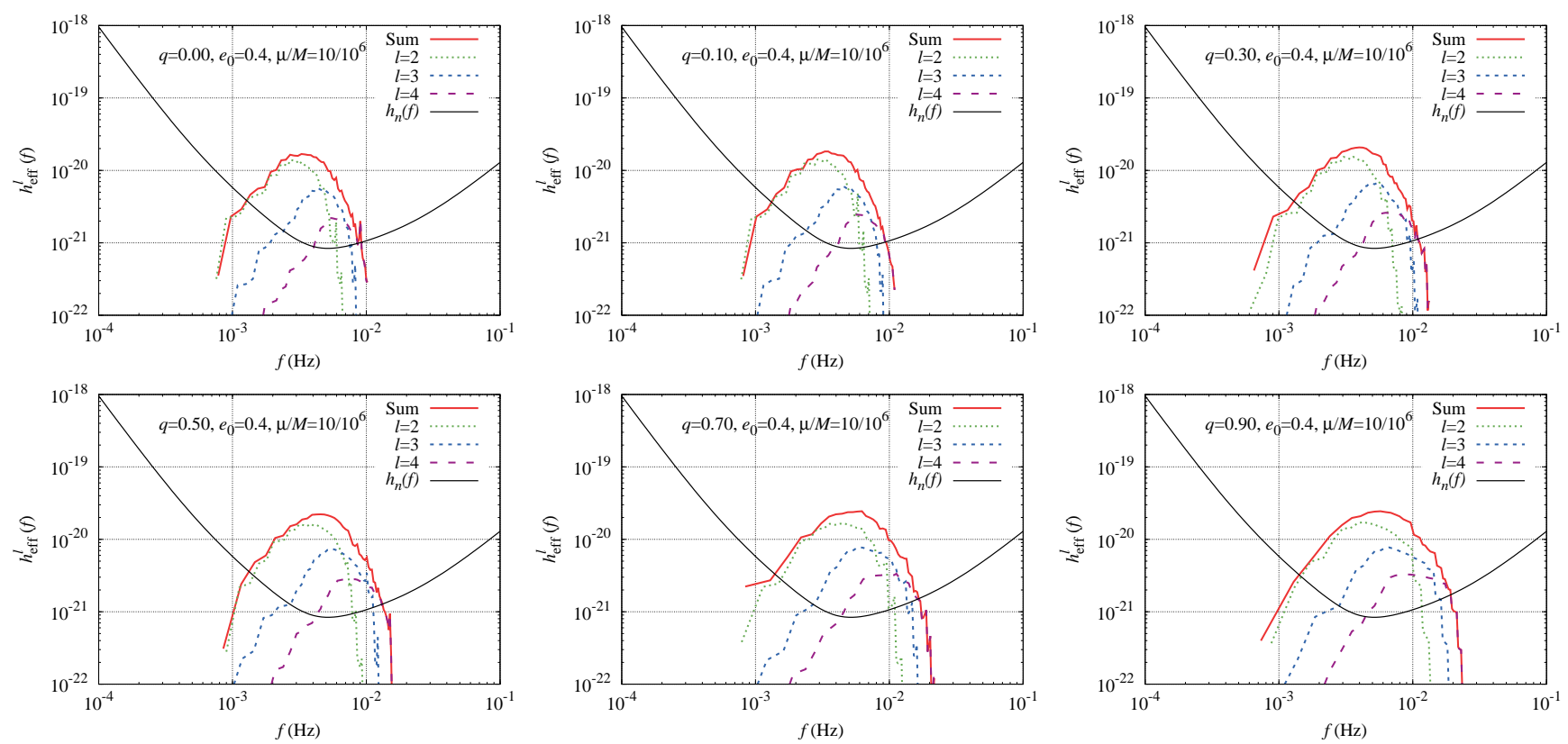

FIG. 10. The same as Fig. 9, but for $e_{0}=0.4$, and $q=0$ (top left), 0.1 (top middle), 0.3 (top right), 0.5 (bottom left), 0.7 (bottom middle), and 0.9 (bottom right). The values of $p_{0}$ take $11.38 M, 11.15 M, 10.71 M, 10.28 M, 9.83 M$, and $9.41 M$ for $q=0,0.1,0.3,0.5,0.7$, and 0.9 , respectively.
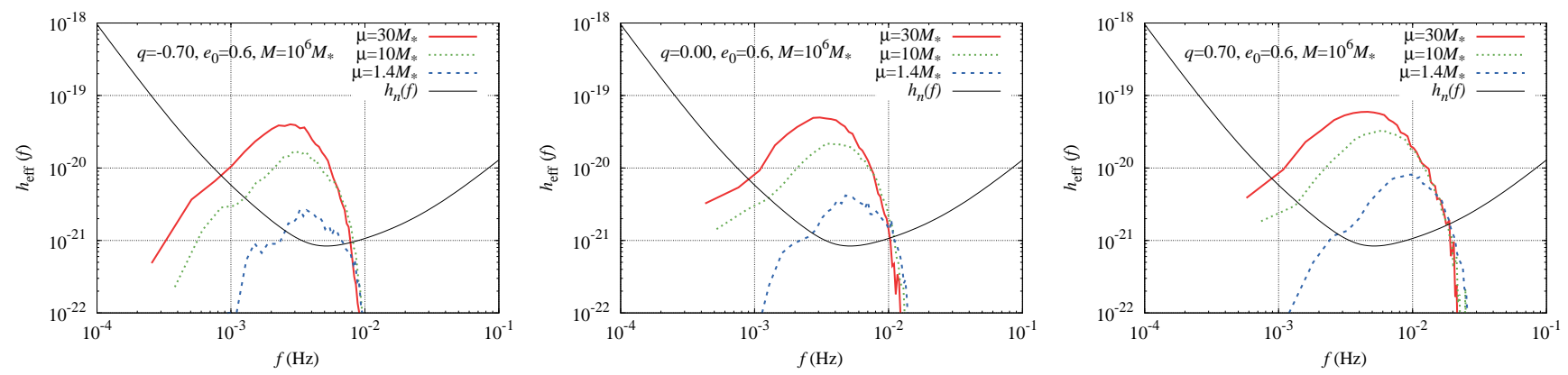

FIG. 11. Power spectrum summed over the $\ell=2-4$ modes for a compact object of mass $\mu=(1.4,10,30) M_{\odot}$ inspiraling around an SMBH of $M=10^{6} M_{\odot}$ with $q=-0.7$ (left), 0 (middle), and 0.7 (right) at $D=1$ Gpc during the last 3 -year inspiral with $e_{0}=0.6$.

However, for larger values of $e_{0}$, the low-frequency tail of gravitational waves (due to the contribution of low- $n$ modes) is above the LISA sensitivity curve and gravitational waves will be detectable by LISA irrespective of $q$ for $M \approx 10^{5} M_{\odot}$ (see also Fig. 16).

In the left panel of Fig. 13 we show the SNR of gravitational waves with respect to the LISA sensitivity curve for $\ell=2-4$ modes for a $10 M_{\odot}$ compact object inspiraling around a $10^{6} M_{\odot} \mathrm{SMBH}$ at $D=1 \mathrm{Gpc}$ during the last 3-year inspiral before plunge. The SNR is plotted as a function of $e_{0}$ for $q=0.5$. It is found that the SNR increases as $e_{0}$ increases because larger number of the $n$-modes could contribute to the SNR. The SNR for the $\ell=2$ mode with $e_{0}=0.8$ is $\approx 80$, which is about 4 times larger than that with $e_{0}=0.1, \approx 20$. Thus, for $M=10^{6} M_{\odot}$, highly eccentric EMRIs could dominate the detection by LISA. The right panel of Fig. 13 shows the SNR as a function of $q$ with $e_{0}=0.4$. As the $\mathrm{BH}$ spin increases, the SNR is significantly increased because the value of $r_{\text {min }}$ near the separatrix decreases and general relativistic effects are enhanced. For example, the SNR for the $\ell=2$ mode with $q=-0.9$ is $\approx 12$ which is about $24 \%$ of the one with $q=0.9, \approx 50$. This indicates that rapidly spinning SMBHs could be more subject to the detection by LISA. However, this is the special feature for $M \gtrsim 10^{6} M_{\odot}$ (see also Fig. 16).

We also note that the SNR for the $\ell=3$ and 4 modes is about $40 \%$ and $20 \%$ of that for the $\ell=2$ mode, respectively. Thus, the detection rate with a template that includes up to the $\ell=3$ and 4 modes of gravitational waves becomes $1.4^{3} \approx 2.7$ and $1.6^{3} \approx 4.1$ times larger than that using only the $\ell=2$ mode, respectively. Obviously, it is crucially important to take into account the high-multipole modes in the waveform modeling. 

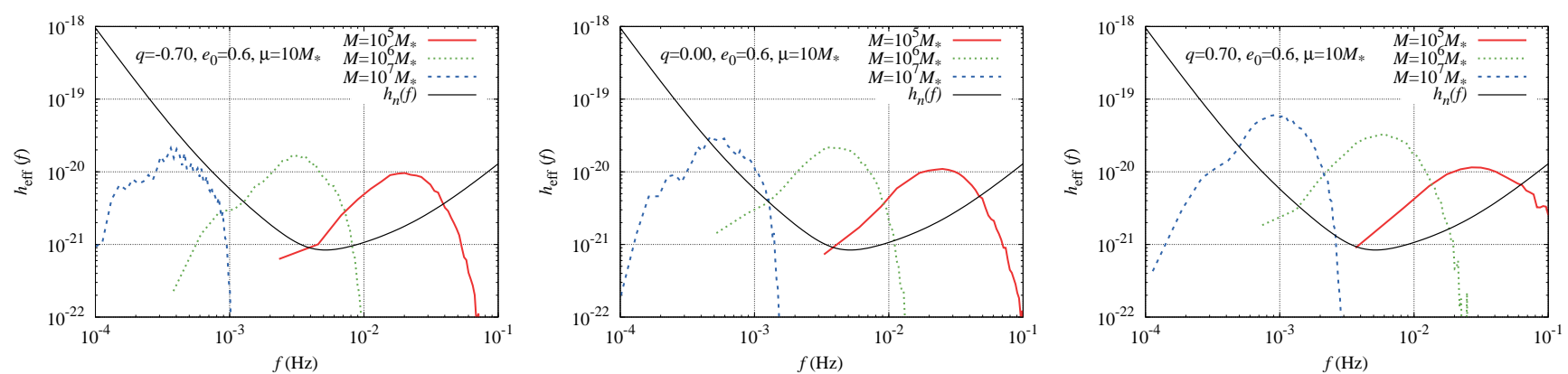

FIG. 12. The same as Fig. 11, but for $\mu=10 M_{\odot}$ and $M=\left(10^{5}, 10^{6}, 10^{7}\right) M_{\odot}$.
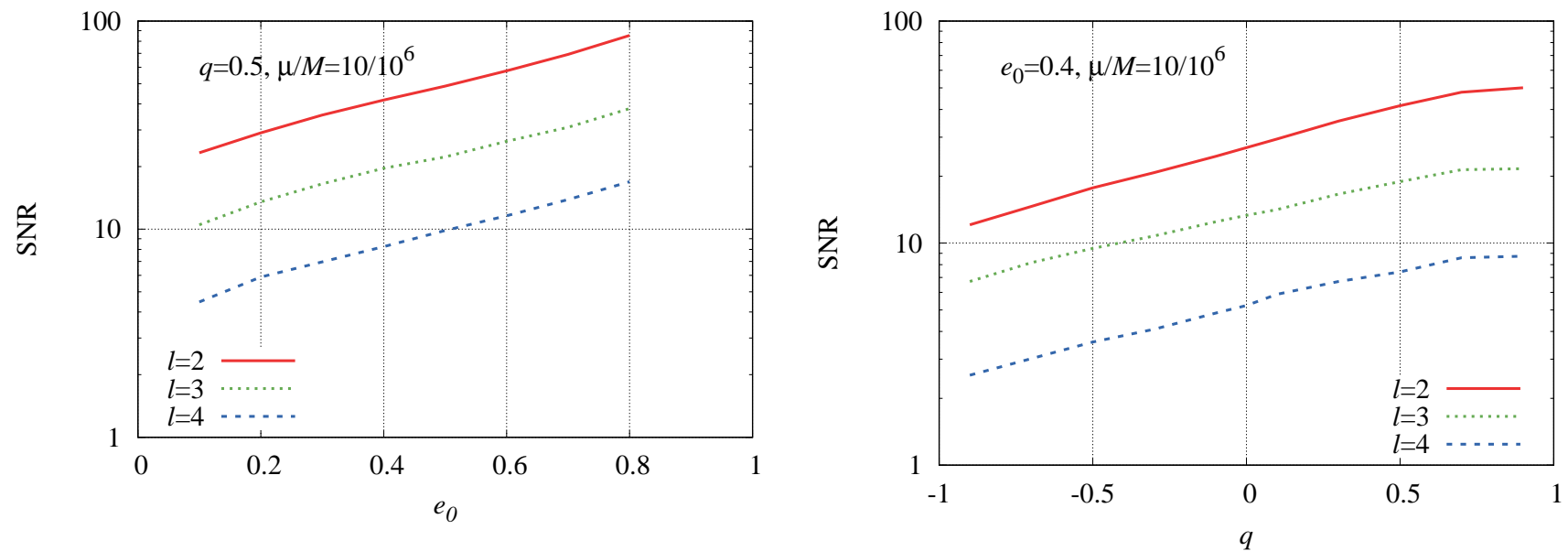

FIG. 13. Left: SNR associated with different $\ell$-modes from $\ell=2$ to 4 as functions of $e_{0}$ for a $10 M_{\odot}$ compact object inspiraling around a $10^{6} M_{\odot} \mathrm{SMBH}$ of spin $q=0.5$ at $D=1 \mathrm{Gpc}$ for the last 3-year inspiral before plunge. Right: SNR as functions of $q$ for $M=10^{6} M_{\odot}, \mu=10 M_{\odot}$, and $e_{0}=0.4$.

Figures 14-16 show the SNR of gravitational waves including the $\ell=2-4$ modes with respect to the LISA designed sensitivity curve for a compact object of mass $\mu$ into an SMBH of mass $M$ at $D=1 \mathrm{Gpc}$ during the last 3-year inspiral before plunge. In Fig. 14, the SNR is shown as a function of $M$ for $\mu=(1.4,10,30) M_{\odot}$ with $e_{0}=0.4$, and $q=-0.5$ (left), 0 (middle), and 0.5 (right). It is found that the SNR is largest for $M \sim 10^{6} M_{\odot}$ irrespective of $q$ and $\mu$, reflecting the sensitivity curve of LISA.

Figure 15 shows the SNR as a function of $e_{0}$ for $M=$ $10^{6} M_{\odot}$ with $q=-0.5$ (left), 0 (middle), and 0.5 (right). As illustrated in Fig. 13, the SNR is a monotonically increasing function of $e_{0}$, that increases by a factor of several for the change from $e_{0}=0.1$ to 0.8 with $M=$ $10^{6} M_{\odot}$. This indicates that highly eccentric EMRIs for this SMBH mass would increase the detection rate in the LISA observation by a factor of several.

Figure 16 shows the SNR as a function of $q$ for $e_{0}=0.4$ with $M=10^{5} M_{\odot}$ (left), $10^{6} M_{\odot}$ (middle), and $10^{7} M_{\odot}$ (right). The SNR increases as the $\mathrm{BH}$ spin increases for $M \gtrsim 10^{6} M_{\odot}$ (see Fig. 13 ), but the SNR for $M=10^{5} M_{\odot}$ depends weakly on $q$ because the late part of the inspirals can be below the LISA frequency band for larger values of $q$ (see Fig. 12). As the right panel of Fig. 16 illustrates, the detection rate of the EMRIs from an SMBH of $M \sim$ $10^{7} M_{\odot}$ depends strongly on the $\mathrm{BH}$ spin: for this $\mathrm{SMBH}$ mass, a higher spin $\mathrm{BH}$ will be much more frequently detected.

\section{Limitation of post-Newtonian formulas}

Before closing Sec. IV, we show the poor accuracy of inspiral orbits determined in the PN approximation by comparing with our numerical results. We use a newly developed $\mathrm{PN}$ formula of $d I^{i} / d t$ that takes into account the PN correction up to $5 \mathrm{PN}$ order and the tenth order in eccentricity. This new formula is the extension of the 4PN formula derived in Ref. [59].

In Fig. 17, we assess the accuracy of the $2 \mathrm{PN}, 3 \mathrm{PN}$, $4 \mathrm{PN}$, and $5 \mathrm{PN}$ formulas using inspiral orbits for $q=$ $-0.5,0$, and 0.5 with $p_{0}=11.4 M$ and $e_{0}=0.2$. Disagreement between the $\mathrm{PN}$ and numerical results is obviously non-negligible. Moreover, the convergence of the PN expansion is quite slow, although with the increase of the PN order the results gradually approach the numerical results. For the numerical inspirals, it takes $\sim 100 M^{2} / \mu$ 

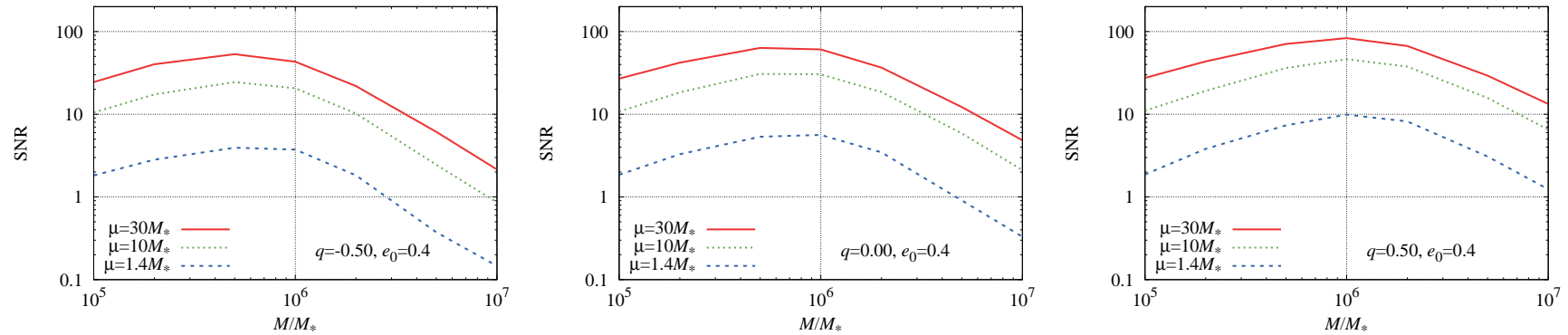

FIG. 14. SNR including the $\ell=2-4$ modes as a function of $M$ for $q=-0.5$ (left), 0 (middle), and 0.5 (right) with $e_{0}=0.4$ and $\mu=(30,10,1.4) M_{\odot}$ for the last 3-year inspiral before plunge.
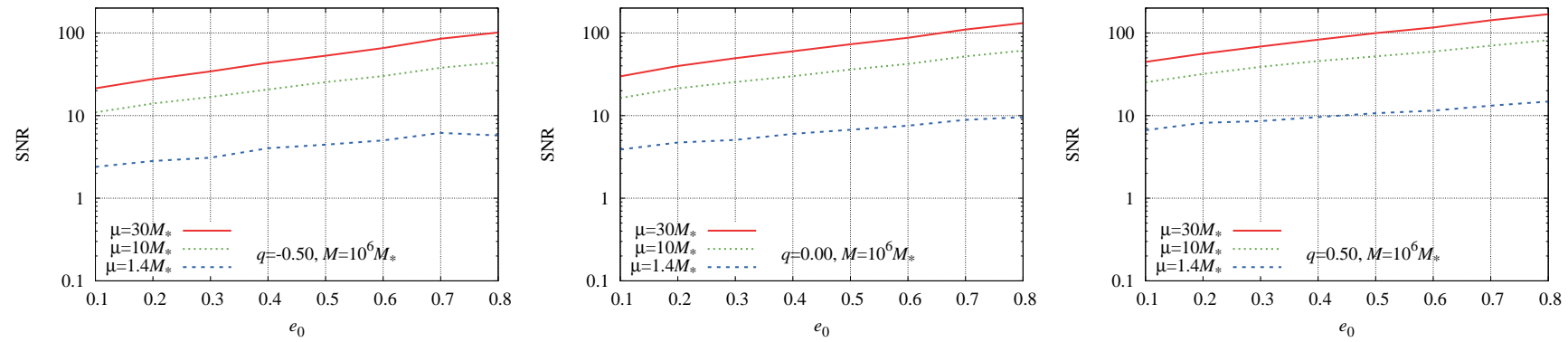

FIG. 15. SNR including the $\ell=2-4$ modes as a function of $e_{0}$ for $q=-0.5$ (left), 0 (middle), and 0.5 (right) with $M=10^{6} M_{\odot}$ and $\mu=(30,10,1.4) M_{\odot}$ for the last 3-year inspiral before plunge.

for $q=-0.5, \sim 190 M^{2} / \mu$ for $q=0$, and $\sim 300 M^{2} / \mu$ for $q=0.5$ until the plunge. On the other hand, for the 5PN inspirals, it takes $\sim 140 \mathrm{M}^{2} / \mu$ for $q=-0.5$, $\sim 210 M^{2} / \mu$ for $q=0$, and $\sim 310 M^{2} / \mu$ for $q=0.5$ until the plunge. The error in the orbital eccentricity at the plunge between the numerical and $5 \mathrm{PN}$ results is about $30 \%$. Thus, the 5PN formulas are not at all accurate enough for gravitational-wave data analysis.

It is interesting to note that the PN formulas work relatively well for $q=0.5$ accidentally. In addition, the $4 \mathrm{PN}$ results often become worse models than the $3 \mathrm{PN}$ results. These facts illustrate that the PN expansion has not only a poor-convergence property but also an irregular convergence property [61,62].

We also note that the convergence of the eccentricity expansion in the PN formulas becomes slower if the eccentricity becomes higher [59]. For $q=0.9$ and $p=6 M$, the relative error of the $5 \mathrm{PN}$ formula in $d E / d t$ determined by the comparison with our numerical results becomes $10^{-3}$ for $e=0.1,10^{-2}$ for $e=0.7$, and $10^{-1}$ for $e=0.9$, although the error is about an order of magnitude smaller than that of the $4 \mathrm{PN}$ and the tenth order in the eccentricity. Thus, the accuracy of inspirals with higher eccentricity becomes worse than that with lower eccentricity.

In Fig. 18, we show the difference in orbital cycles using the PN and numerical results. This clearly illustrates the poor-convergence property and limitation in the PN formulas because the difference in the orbital cycles from the numerical results is of the order of $10^{3}$ even for the $5 \mathrm{PN}$ formula, in spite of the fact that the required accuracy is within 0.1 orbital cycles. These results agree with those in Refs. [63, 64], which study quasicircular inspirals. To conclude, the $5 \mathrm{PN}$ formulas, which are currently the best analytic ones, cannot be used in the original forms for the data analysis of gravitational waves.

To derive an accurate analytic or semianalytic formula, the PN formula combined with other methods such as resummation methods and numerical fitting methods of higher PN order coefficients are inevitable [65-75]. However, a significant improvement is required. In addition, new ideas would be necessary for eccentric orbits because we need to perform a resummation or fitting with respect not only to the PN expansion parameter (e.g., $(M / p)^{1 / 2}$ ) but also to the eccentricity, $e$. In particular, no idea for an efficient resummation with respect to the eccentricity has been proposed. We encourage the readers to perform a careful analysis of our numerical data for developing a novel scheme of a resummation/numerical fitting. Our numerical data and 5PN formulas are published in a web site [76].

\section{SUMMARY}

We computed gravitational waves from a stellar-mass compact object inspiraling around an SMBH. The inspiral orbits were determined by taking into account the 

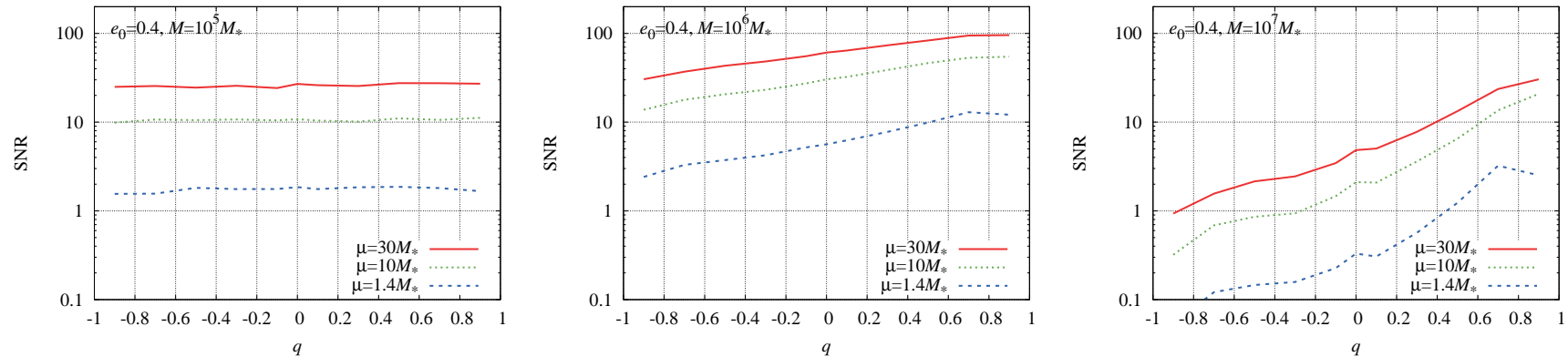

FIG. 16. SNR including the $\ell=2-4$ modes as functions of $q$ for $M=10^{5} M_{\odot}$ (left), $10^{6} M_{\odot}$ (middle), and $10^{7} M_{\odot}$ (right) with $\mu=(30,10,1.4) M_{\odot}$ for the last 3-year inspiral before plunge.
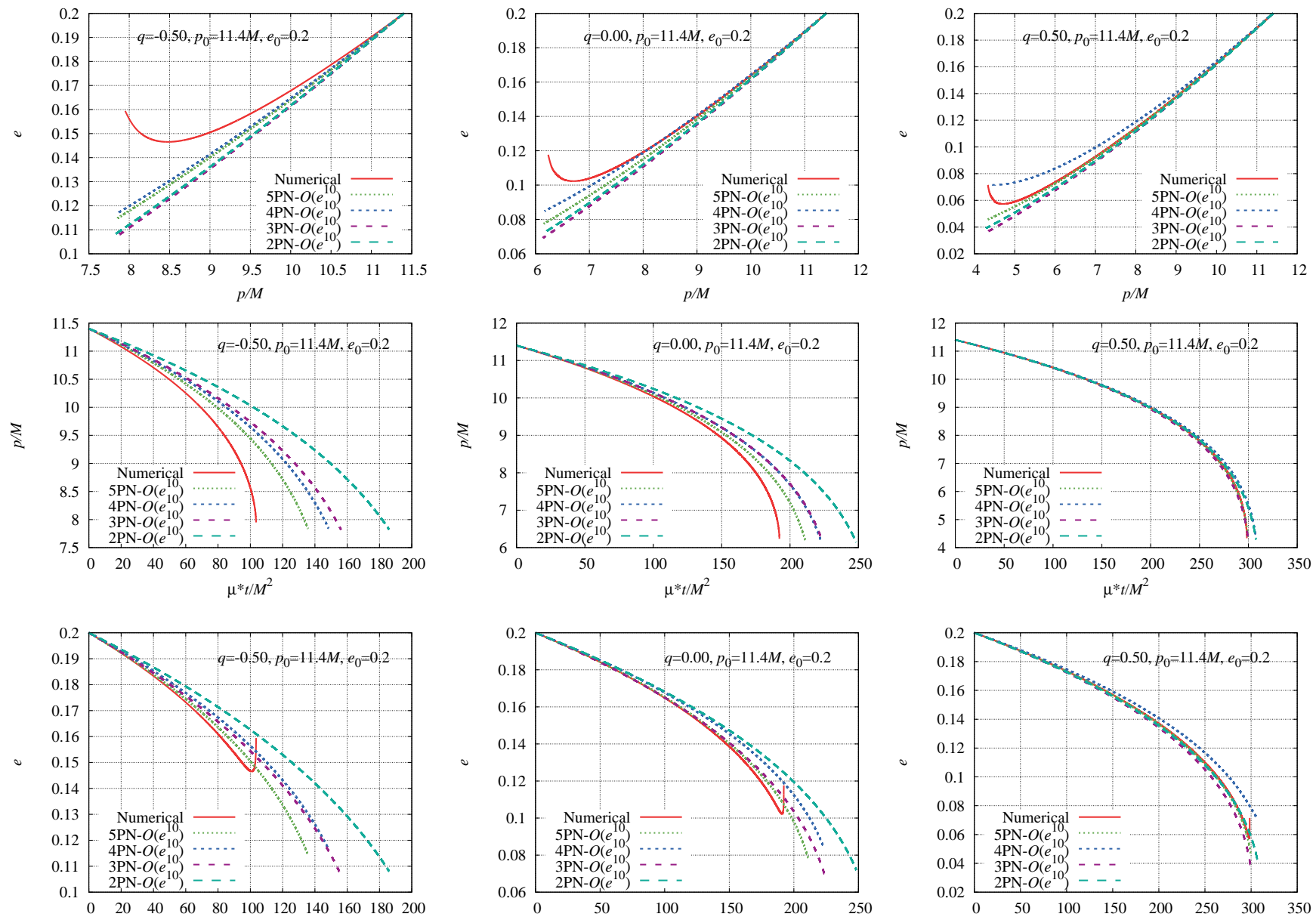

FIG. 17. Orbital evolution using numerical fluxes and PN fluxes for $q=-0.5$ (left), 0 (middle), and 0.5 (right) with $p_{0}=11.4 M$ and $e_{0}=0.2$. Top panels show $e$ as a function of $p / M$, middle panels show $p / M$ as a function of $\mu t / M^{2}$, and bottom panels show $e$ as a function of $\mu t / M^{2}$.

adiabatic change of the constants of motion, $d I^{i} / d t$, due to the emission of gravitational waves. In our procedure, we first obtained $d I^{i} / d t$ for $\approx 2 \times 10^{4}$ data points in the parameter space of $(p, e)$ for each value of $q$. Then, accurate interpolation was used to derive gravitational-wave fluxes at arbitrary points within the region of the parameter space computed in advance. The relative error in the interpolated values of $d I^{i} / d t$ is typically $\lesssim 10^{-6}$, which is smaller than the inverse of the gravitational-wave phase for EMRIs during the last 3-year observation in LISA, for most of the parameter space except for $r_{\min } \lesssim 3 M$ (see Sec. III).

In Sec. IV, we derived the inspiral orbits and associated gravitational waves. We then computed the spectrum of 

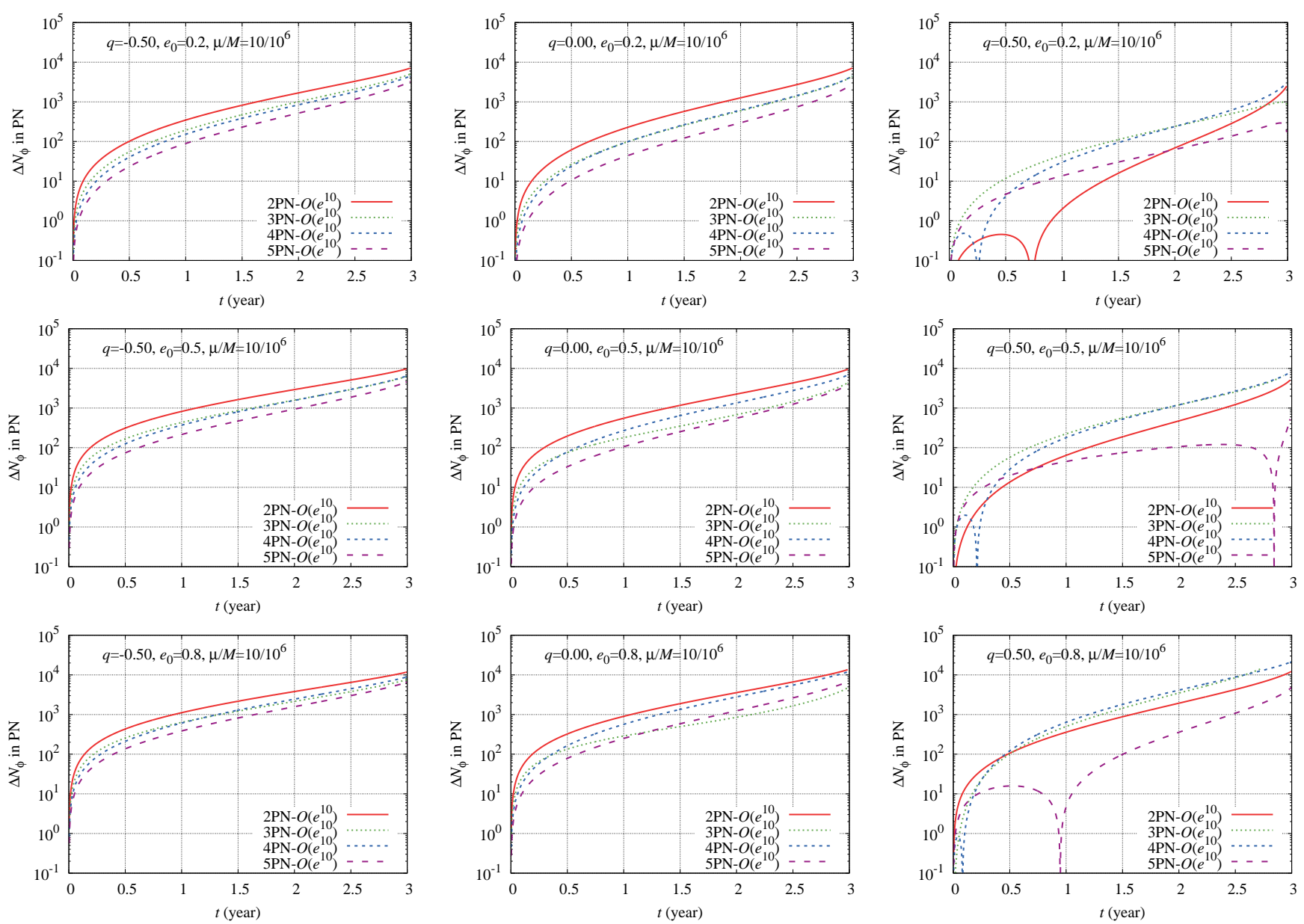

FIG. 18. Difference in orbital cycle for the last 3-year inspirals computed using numerical and PN fluxes for $q=-0.5$ (left), 0 (middle), and 0.5 (right) with $e_{0}=0.2$ (top), 0.5 (middle), and 0.8 (bottom), and $(M, \mu)=\left(10^{6}, 10\right) M_{\odot}$.

gravitational waves and the SNR for several values of mass of a binary, the $\mathrm{BH}$ spin, and the initial orbital eccentricity during the 3-year LISA observation before final plunge. We found that the SNR increases by a factor of several as the $\mathrm{BH}$ spin and the mass of the compact object increase for $M \gtrsim 10^{6} M_{\odot}$. The SNR as a function of the $\mathrm{BH}$ mass has a maximum around $M=10^{6} M_{\odot}$ for fixed values of $q$ and $e_{0}$. The SNR as a function of $q$ is weakly dependent on $M$ around $M=10^{5} M_{\odot}$ because only an early part of the inspirals can be observed in the LISA frequency band for the larger $\mathrm{BH}$ spin. The SNR as a function of the initial orbital eccentricity for $M=10^{6} M_{\odot}$ is a monotonically increasing function, that increases by a factor of several for the change from $e_{0}=$ 0.1 to 0.8 . We also found that the SNR for the $\ell=3(\ell=$ 4) modes is about $40 \%$ (20\%) of that for the $\ell=2$ mode. This shows that taking account of the higher multipole modes of gravitational waves is important for increasing the detection rate in the LISA observation by a factor of $3-4$.

In Sec. IV C, the limitation of the PN formulas is shown by comparing the orbital cycles between the numerical and PN inspirals. The difference in the orbital cycles becomes larger than $10^{3}$ even for the $5 \mathrm{PN}$ formula, which is much larger than the required accuracy in the LISA data analysis, $\lesssim 1 \mathrm{rad}$ in phase. This illustrates that we need much higher-order PN formulas or to develop a special prescription such as resummation to improve the accuracy in the PN formula.

In our present numerical computation, the numerical accuracy of the gravitational fluxes for compact orbits with $r_{\text {min }} \lesssim 3 M$ is not high enough. Such compact orbits are possible for a high value of $q \gtrsim 0.6$. As we showed in Sec. IVB, the SNR is higher for higher spin SMBHs with mass $M \approx 10^{6}-10^{7} M_{\odot}$, and hence, the detectability of EMRIs for the relatively high-mass SMBHs will be higher for the higher spin SMBH. This indicates that it is important to develop accurate gravitationalwave models for the high values of $q$. As we discussed in Sec. III, the accuracy could be straightforwardly improved if we could perform the computation with higher numerical precision. A question is how high numerical precision is required for each value of $q$. This is one of our next issues to be clarified. 
In general, orbital inspirals of a compact object into an SMBH are not only eccentric but also inclined from the equatorial plane of the SMBH. Thus, it is necessary to extend our approach to eccentric and inclined inspirals. The semilatus rectum at separatrix becomes larger for larger orbital inclination angle with fixed orbital eccentricity and $\mathrm{BH}$ spin. This implies that orbital inclination effectively reduces the effects of the $\mathrm{BH}$ spin and the frequency of gravitational waves at separatrix. We expect the power spectra of gravitational waves and SNR for eccentric and inclined inspirals in LISA observation would be smaller than those for equatorial inspirals studied in this paper. To check this quantitatively, we need to compute gravitational waves for a large set of parameter space in the $\mathrm{BH}$ spin, the semilatus rectum, the eccentricity, and the inclination angle from the equatorial plane of the $\mathrm{BH}$. It would take about a year to derive gravitational waves for $\sim 10^{6}$ points in $\left(q, p, e, \theta_{\text {inc }}\right)$ with $q \lesssim 0.9$ and $e \lesssim 0.9$ using a $\sim 10$ Tflops machine if it takes 10 times longer to compute gravitational waves for a nonequatorial orbit than the one for an equatorial orbit (see Sec. III). However, it is not clear how many data points are necessary to accurately derive inspiral orbits for the generic case by interpolation methods. We are currently working on this issue, and the results will be published in future.

\section{ACKNOWLEDGMENTS}

We would like to thank the anonymous referee for useful comments and suggestions. This work was in part supported by JSPS/Ministry of Education, Culture, Sports, Science and Technology (MEXT) KAKENHI Grants No. JP16H02183, No. JP18H04583, and No. JP20H00158.
[1] P. Amaro-Seoane et al. (LISA Collaboration), arXiv:1702.00786.

[2] J. Luo et al. (TianQin Collaboration), Classical Quantum Gravity 33, 035010 (2016).

[3] W. H. Ruan, Z. K. Guo, R. G. Cai and Y. Z. Zhang, Int. J. Mod. Phys. A. 35, 2050075 (2020).

[4] S. Babak et al., Phys. Rev. D 95, 103012 (2017).

[5] C. P. L. Berry et al., arXiv:1903.03686.

[6] Y. Mino, M. Sasaki, M. Shibata, H. Tagoshi and T. Tanaka, Prog. Theor. Phys. Suppl. 128, 1 (1997).

[7] M. Sasaki and H. Tagoshi, Living Rev. Relativity 6, 6 (2003).

[8] Y. Mino, M. Sasaki and T. Tanaka, Phys. Rev. D 55, 3457 (1997).

[9] T. C. Quinn and R. M. Wald, Phys. Rev. D 56, 3381 (1997).

[10] E. Poisson, A. Pound and I. Vega, Living Rev. Relativity 14, 7 (2011).

[11] L. Barack, Classical Quantum Gravity 26, 213001 (2009).

[12] L. Barack and A. Pound, Rep. Prog. Phys. 82, 016904 (2019).

[13] T. Hinderer and E. E. Flanagan, Phys. Rev. D 78, 064028 (2008).

[14] E. E. Flanagan and T. Hinderer, Phys. Rev. Lett. 109, 071102 (2012).

[15] M. Shibata, Prog. Theor. Phys. 90, 595 (1993).

[16] S. A. Hughes, Phys. Rev. D 61084004 (2000).

[17] S. A. Hughes, Phys. Rev. D 64064004 (2001).

[18] T. Tanaka, M. Shibata, M. Sasaki, H. Tagoshi and T. Nakamura, Prog. Theor. Phys. 90, 65 (1993).

[19] C. Cutler, D. Kennefick and E. Poisson, Phys. Rev. D 50, 3816 (1994).

[20] K. Glampedakis and D. Kennefick, Phys. Rev. D 66 044002 (2002).

[21] S. Drasco and S. A. Hughes, Phys. Rev. D 73, 024027 (2006); 88, 109905(E) (2013); 90, 109905(E) (2014).

[22] S. Drasco, Phys. Rev. D 79, 104016 (2009).

[23] R. Fujita, W. Hikida and H. Tagoshi, Prog. Theor. Phys. 121, 843 (2009).
[24] A. Pound and E. Poisson, Phys. Rev. D 77, 044013 (2008).

[25] J. R. Gair, E. E. Flanagan, S. Drasco, T. Hinderer and S. Babak, Phys. Rev. D 83, 044037 (2011).

[26] N. Warburton, S. Akcay, L. Barack, J. R. Gair and N. Sago, Phys. Rev. D 85, 061501 (2012).

[27] T. Osburn, N. Warburton and C. R. Evans, Phys. Rev. D 93, 064024 (2016).

[28] R. Fujita and H. Tagoshi, Prog. Theor. Phys. 112, 415 (2004).

[29] R. Fujita and H. Tagoshi, Prog. Theor. Phys. 113, 1165 (2005).

[30] S. Mano, H. Suzuki and E. Takasugi, Prog. Theor. Phys. 95, 1079 (1996).

[31] S. Mano, H. Suzuki and E. Takasugi, Prog. Theor. Phys. 96, 549 (1996).

[32] S. Mano and E. Takasugi, Prog. Theor. Phys. 97, 213 (1996).

[33] Y. Mino, Phys. Rev. D 67084027 (2003).

[34] J. M. Bardeen, W. H. Press and S. A. Teukolsky, Astrophys. J. 178, 347 (1972).

[35] W. Schmidt, Classical Quantum Gravity 19, 2743 (2002).

[36] R. Fujita and W. Hikida, Classical Quantum Gravity 26, 135002 (2009).

[37] S. Drasco and S. A. Hughes, Phys. Rev. D 69, 044015 (2004).

[38] S. A. Teukolsky, Astrophys. J. 185, 635 (1973).

[39] N. Sago, T. Tanaka, W. Hikida and H. Nakano, Prog. Theor. Phys. 114509 (2005).

[40] N. Sago, T. Tanaka, W. Hikida, K. Ganz and H. Nakano, Prog. Theor. Phys. 115873 (2006).

[41] S. A. Teukolsky and W. H. Press, Astrophys. J. 193443 (1974).

[42] S. Drasco, E. E. Flanagan and S. A. Hughes, Classical Quantum Gravity 22, S801 (2005).

[43] W. H. Press, S. A. Teukolsky, W. T. Vetterling and B. P. Flannery, Numerical Recipes in $C$ (Cambridge University Press, Cambridge, United Kingdom, 1992). 
[44] A. G. Shah, J. L. Friedman and B. F. Whiting, Phys. Rev. D 89, 064042 (2014).

[45] A. G. Shah, Phys. Rev. D 90, 044025 (2014).

[46] A. G. Shah and A. Pound, Phys. Rev. D 91, 124022 (2015).

[47] S. Hopper, E. Forseth, T. Osburn and C. R. Evans, Phys. Rev. D 92, 044048 (2015).

[48] P. C. Peters, Phys. Rev. 136, B1224 (1964).

[49] P. Amaro-Seoane, C. F. Sopuerta, and M. D. Freitag, Mon. Not. R. Astron. Soc. 429, 3155 (2013).

[50] L. S. Finn and K. S. Thorne, Phys. Rev. D 62, 124021 (2000).

[51] C. J. Moore, R. H. Cole and C. P. L. Berry, Classical Quantum Gravity 32, 015014 (2015).

[52] J. R. Gair and K. Glampedakis, Phys. Rev. D 73, 064037 (2006).

[53] S. Babak, H. Fang, J. R. Gair, K. Glampedakis and S. A. Hughes, Phys. Rev. D 75, 024005 (2007); 77, 04990(E) (2008).

[54] A. J. K. Chua, C. J. Moore, and J. R. Gair, Phys. Rev. D 96, 044005 (2017).

[55] L. Barack and C. Cutler, Phys. Rev. D 69, 082005 (2004).

[56] Black Hole Perturbation Toolkit, https://bhptoolkit.org/.

[57] T. A. Apostolatos, C. Cutler, G. J. Sussman and K. S. Thorne, Phys. Rev. D 49, 6274 (1994).

[58] L. Blanchet, Living Rev. Relativity 17, 2 (2014).

[59] N. Sago and R. Fujita, Prog. Theor. Exp. Phys. 2015, 073E03 (2015).

[60] P. C. Peters and J. Mathews, Phys. Rev. 131, 435 (1963).
[61] N. Sago, R. Fujita and H. Nakano, Phys. Rev. D 93, 104023 (2016).

[62] R. Fujita, N. Sago and H. Nakano, Classical Quantum Gravity 35, 027001 (2018).

[63] R. Fujita, Prog. Theor. Phys. 128, 971 (2012).

[64] R. Fujita, Prog. Theor. Exp. Phys. 2015, 033E01 (2015).

[65] T. Damour, B. R. Iyer and B. S. Sathyaprakash, Phys. Rev. D 57, 885 (1998).

[66] A. Buonanno and T. Damour, Phys. Rev. D 59, 084006 (1999).

[67] A. Buonanno and T. Damour, Phys. Rev. D 62, 064015 (2000).

[68] T. Damour, B. R. Iyer and B. S. Sathyaprakash, Phys. Rev. D 63, 044023 (2001); 72, 029902(E) (2005).

[69] T. Damour and A. Nagar, Phys. Rev. D 76, 064028 (2007)

[70] T. Damour and A. Nagar, Phys. Rev. D 77, 024043 (2008).

[71] T. Damour, B. R. Iyer and A. Nagar, Phys. Rev. D 79, 064004 (2009).

[72] A. Buonanno, B. Iyer, E. Ochsner, Y. Pan and B. S. Sathyaprakash, Phys. Rev. D 80, 084043 (2009).

[73] N. Yunes, A. Buonanno, S. A. Hughes, M. Coleman Miller and Y. Pan, Phys. Rev. Lett. 104, 091102 (2010).

[74] N. Yunes, A. Buonanno, S. A. Hughes, Y. Pan, E. Barausse, M. C. Miller and W. Throwe, Phys. Rev. D 83, 044044 (2011); 88, 109904(E) (2013).

[75] S. Isoyama, R. Fujita, N. Sago, H. Tagoshi and T. Tanaka, Phys. Rev. D 87, 024010 (2013).

[76] Black Hole Perturbation Club, https://sites.google.com/view/bhpc1996/home. 